FedUni ResearchOnline

http://researchonline.federation.edu.au

This is the peer-reviewed version of the following article:

Vamplew, P., et al. (2017) Softmax exploration strategies for multiobjective reinforcement learning. Neurocomputing Vol. 263, p. 74-86.

Which has been published in final form at:

https://doi.org/10.1016/j.neucom.2016.09.141

Copyright $(2017$ Elsevier B.V. All rights reserved. 


\title{
Softmax Exploration Strategies for Multiobjective Reinforcement Learning
}

\author{
Peter Vamplew, Richard Dazeley and Cameron Foale \\ Federation Learning Agents Group, School of Engineering and Information Technology, \\ Federation University Australia, Ballarat, Victoria, Australia
}

\begin{abstract}
Despite growing interest over recent years in applying reinforcement learning to multiobjective problems, there has been little research into the applicability and effectiveness of exploration strategies within the multiobjective context. This work considers several widely-used approaches to exploration from the single-objective reinforcement learning literature, and examines their incorporation into multiobjective Q-learning. In particular this paper proposes two novel approaches which extend the softmax operator to work with vectorvalued rewards. The performance of these exploration strategies is evaluated across a set of benchmark environments. Issues arising from the multiobjective formulation of these benchmarks which impact on the performance of the exploration strategies are identified. It is shown that of the techniques considered, the combination of the novel softmax-epsilon exploration with optimistic initialisation provides the most effective trade-off between exploration and exploitation.
\end{abstract}

Keywords: multiobjective reinforcement learning, exploration, $\epsilon$-greedy exploration, optimistic initialisation, softmax

\section{Introduction}

Most reinforcement learning (RL) algorithms consider only a single objective, encoded in a scalar reward. However, many sequential decision-making tasks naturally have multiple conflicting objectives, such as minimising both distance and risk of congestion in path-finding [1,2], optimizing both energy efficiency and quality of communication in wireless networks [3], and control tasks with multiple performance indices [4]. Therefore recent years have seen 
multiobjective reinforcement learning (MORL) emerge as a growing area of research [5]. One issue which has yet to be investigated to any significant extent is the role of exploration in a multiobjective context. Balancing the exploitation of the agent's current learning against the potential to improve the current policy through exploratory actions is critical to the performance of a RL agent, particularly in online learning [6]. While there has recently been extensive examination of this trade-off for multiobjective multi-armed bandits (e.g. [7, 8]), there has been no work yet addressing exploration in general multiobjective environments with multiple states.

This paper considers three approaches to exploration which have been widely used in the single-objective reinforcement learning literature ( $\epsilon$-greedy exploration, softmax exploration and optimistic initialisation), and examines how they can be applied in the context of multiobjective reinforcement learning. The three methods are incorporated into a multiobjective formulation of the $\mathrm{Q}(\lambda)$ learning algorithm, which in some cases requires modifications to aspects of the exploration algorithm, and then evaluated across three benchmark multiobjective environments. The results of these empirical evaluations demonstrate that exploration in a multiobjective environment differs from exploration in single-objective reinforcement learning, and provide insight into the correct choice of exploration strategy and settings.

\section{Background}

This section of the paper provides the necessary background information on multiobjective reinforcement learning (and specifically value-based approaches to MORL), and single-objective exploration methods. Section 3 will build on this background to explore what modifications, if any, are required to adapt these exploration methods to apply to multiobjective RL.

\subsection{Multiobjective Reinforcement Learning}

The fundamental difference between single-objective and multiobjective $\mathrm{RL}$ is that the latter has vector-valued rewards (with each element of the vector corresponding to one of the objectives) rather than scalar rewards. Value-based approaches to RL can therefore be extended to MORL by simply storing a vector value for each state-action pair. That is, instead of the value of an action $a$ in a state $s$ being presented by a scalar $Q(s, a)$, it is represented by the vector value $\bar{Q}(s, a)$. For example Algorithm 1 outlines how Q-learning can be extended to handle multiple objectives. Note that in this algorithm 
and for the remainder of the paper, $Q(s, a, o)$ denotes the scalar value of action $a$ in state $s$ with respect to objective $o, \bar{Q}(s, a)$ denotes the vector value of action $a$ in state $s$ for all objectives, and $\bar{Q}(s)$ denotes the vector values of all actions in state $s$.

The primary issue is then how to perform action selection to form a policy on the basis of these vector values, as required by lines 9,12 and 13 of Algorithm 1. In single-objective RL, one or more actions will have a $Q(s, a)$ value which exceeds or equals the value of all other actions, and so the greedy policy simply selects one of these actions. However when the $Q(s, a)$ values are vectors action selection is no longer as simple, as one action may be maximal for one objective, and a different action be optimal with regards to another objective. Formally, there may be multiple actions which are non-dominated in terms of the Pareto-dominance relationship [5]. Therefore the agent must incorporate an action-selection function which defines an ordering over these vector values, so as to allow the greedy action to be identified as shown in Equation 1. $U$ has been used to represent this actionselection function in reference to the concept of utility which forms the basis for economic theories of preference [9].

$$
U(\bar{Q}(s, a), \bar{Q}(s, b))= \begin{cases}+1, & \text { if } a \text { is preferred to } b(\bar{Q}(s, a) \succ \bar{Q}(s, b)) \\ -1, & \text { if } b \text { is preferred to } a(\bar{Q}(s, a) \prec \bar{Q}(s, b)) \\ 0, & \text { otherwise }(\bar{Q}(s, a) \sim \bar{Q}(s, b))\end{cases}
$$

Commonly action selection is implemented via a scalarisation function $f$ which maps the vectors to scalar values [10], as shown in Equation 2.

$$
U_{\text {scalar }}(\bar{Q}(s, a), \bar{Q}(s, b))= \begin{cases}+1, & \text { if } f(\bar{Q}(s, a))>f(\bar{Q}(s, b)) \\ -1, & \text { if } f(\bar{Q}(s, a))<f(\bar{Q}(s, b)) \\ 0, & \text { if } f(\bar{Q}(s, a))=f(\bar{Q}(s, b))\end{cases}
$$

The scalarisation function is often parameterised so that the desired trade-offs between objectives can be expressed via these parameters [5]. One approach to scalarisation is to calculate a linear weighted sum of the objective values as shown in Equation 3, where the weights $w_{o}$ indicate the relative importance of the objectives:

$$
f(\bar{Q}(s, a))=\sum_{o=1}^{n} w_{o} Q(s, a, o)
$$




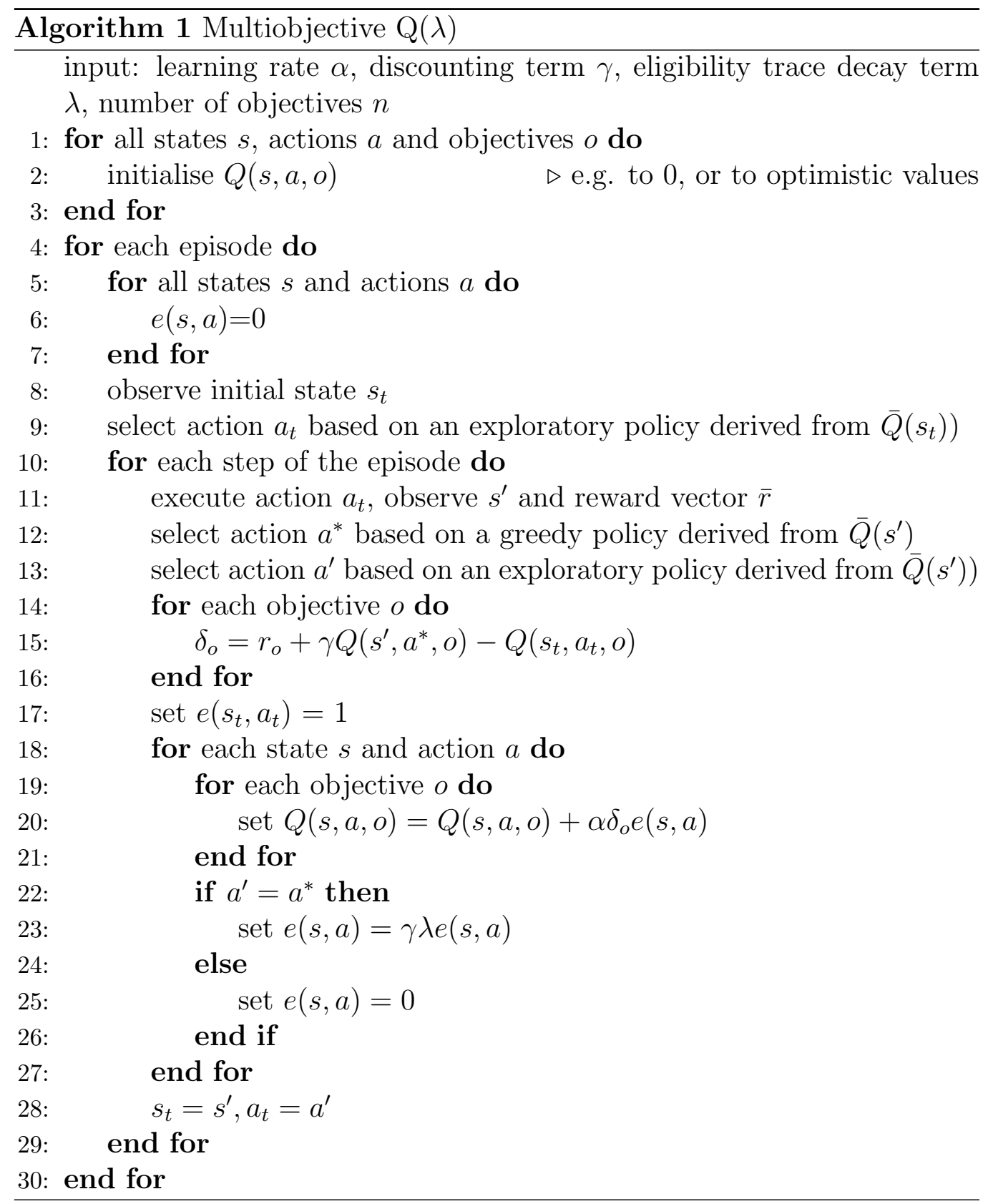


Linear scalarisation is simple to implement and remains the most widely used form of MORL, particularly in applied research (e.g. [11, 12]). However there are contexts in which it can not adequately express the desired trade-off between objectives [5] or discover the most appropriate policy [13].

Consequently several researchers have examined the use of non-linear approaches to action selection, such as thresholded lexicographic ordering [14], the hypervolume metric [15], and Chebyshev distance [16]. These non-linear approaches can be a more appropriate means of specifying the required tradeoffs between objectives for many problems. For example, thresholded lexicographic ordering is a natural means of specifying the desire to maximise one objective subject to achieving threshold levels of performance on the other objective(s).

As noted by [5], linear scalarisation of the reward vector essentially converts the problem from a multiobjective Markov Decision Process (MOMDP) into a more conventional single-objective MDP. Therefore exploration methods will function in the same manner as they do for single-objective RL. Hence the more interesting case is the application of exploration methods to MORL in conjunction with non-linear approaches to action selection. For the purposes of this study we will focus on the thresholded lexicographic ordering (TLO) approach to action-selection as this is the most widely used non-linear approach [14, 17, 18, 19]. However the methods developed and tested within this study are equally applicable to other forms of non-linear action selection.

The foundation of TLO is a lexicographic ordering of vectors as defined in Algorithm 2. TLO simply applies a thresholding operation to each element of the vectors $\bar{A}$ and $\bar{B}$ prior to performing lexicographic comparison, as shown in Algorithm 3. Hence the aim of an agent using TLO-based action selection is to maximise the value of objective $n$, subject to satisfying the constraints of achieving threshold values for the other objectives. By comparing the Q-values of all pairs of actions available in the current state, an agent can identify the greedy action to select. In many problems the threshold values arise from a definition of the problem and the desired outcome for example, an agent controlling a self-driving vehicle may aim to minimise travel time, subject to keeping the risk of an accident below an acceptable level. Alternatively, as will be the case in the experiments reported in this paper, an approximation to the complete Pareto front of policies might be achieved by repeatedly executing a single-policy learning algorithm based on TLO with different sets of threshold values. 

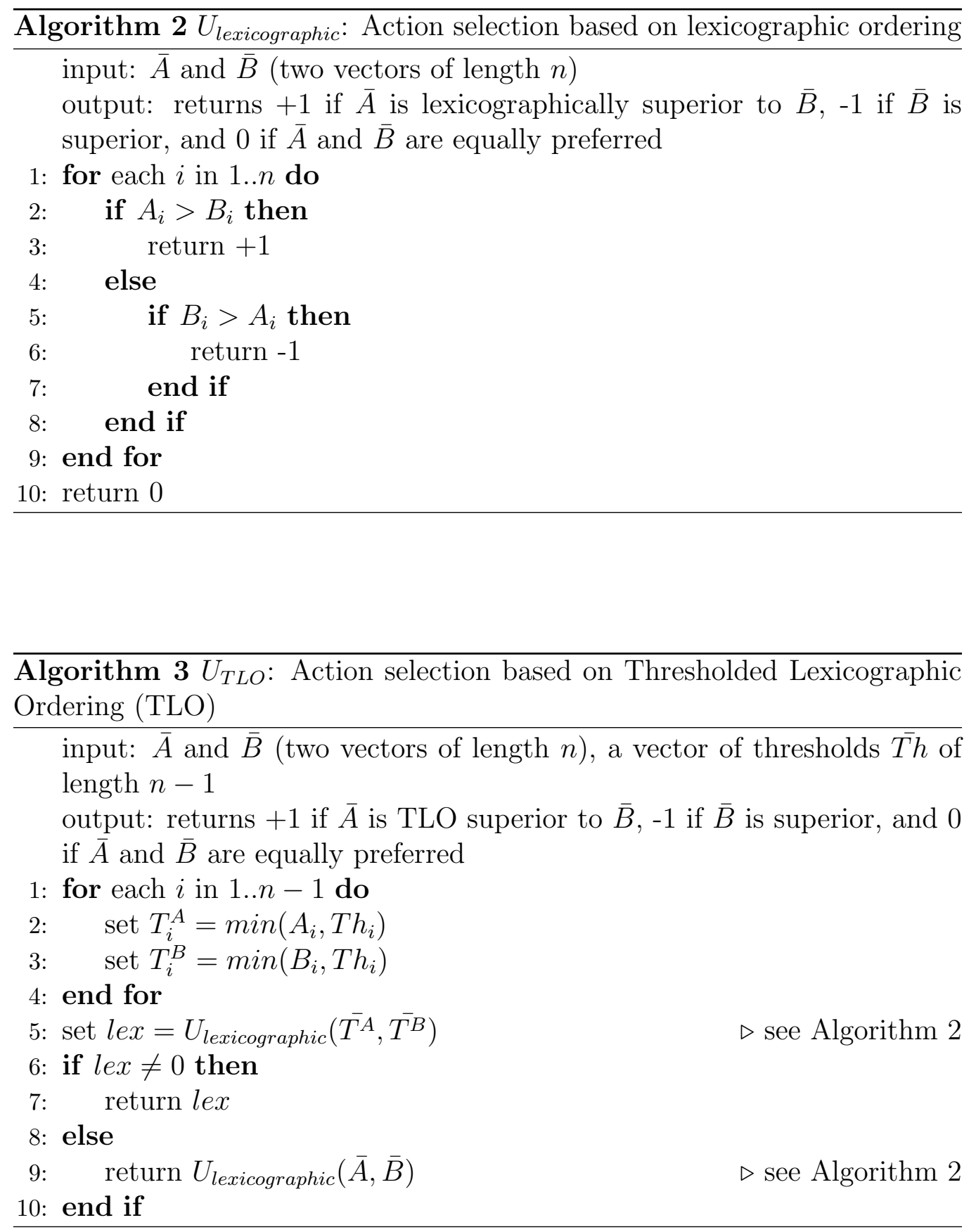


\subsection{Exploration in Single-Objective $R L$}

As noted in the introduction, the tradeoff between exploration and exploitation is a critical factor in the performance of a RL agent. Value-based RL methods require that all states and actions are experienced sufficiently often to allow the true value function to be learnt. An insufficient level of exploration may lead to learning of a sub-optimal policy. However overexploration can lead to poor performance during learning, which may be unacceptable, particularly when learning in an online situation. While this exploration-exploitation tradeoff in single-objective RL has been widely studied (for example, $[20,21,22]$ ) in practice three relatively simple approaches remain widely used. These are $\epsilon$-greedy exploration, softmax exploration and optimistic initialisation.

An $\epsilon$-greedy strategy, at each time step, either selects the greedy action with probability $1-\epsilon$, or selects an action at random with probability $\epsilon$. The value of $\epsilon$ is generally decayed over time to 0 so that the agent eventually converges to following a strictly greedy policy. The exploitation-exploration trade-off is controlled via the initial value of $\epsilon$, with higher values resulting in a greater emphasis on exploration. The primary limitation of this approach is its randomness - all non-greedy actions are treated equally regardless of how likely it is that they might in fact yield an improvement in policy.

Softmax exploration provides a somewhat more sophisticated approach by taking into account the relative values of all actions. The probability of selection of each action is determined by Equation 4 .

$$
P(a)=\frac{e^{Q(s, a) / \tau}}{\sum_{b=1}^{n} e^{Q(s, b) / \tau}}
$$

As with $\epsilon$-greedy exploration, the extent to which exploration takes place is controlled by a single parameter - in this case the temperature or $\tau$ parameter. At high temperatures all actions will be selected with almost equal probability regardless of their estimated value. The temperature is generally reduced during learning, leading the agent to increasingly focus on highervalued actions. As the temperature approaches zero, the agent's choice of action converges towards a strictly greedy policy. The primary criticism of softmax is that the setting of the temperature parameter is unintuitive, particularly in comparison to the straightforward interpretation of $\epsilon$ in $\epsilon$-greedy exploration.

Another commonly-used approach to producing more directed exploration 
is via optimistic initialisation of state-action values. By setting the initial values to be higher than their expected final values, actions which have been relatively infrequently executed will tend to be valued more highly than other actions in the same state. In this way the agent is encouraged to perform a broad exploration of the states and actions of the environment even when selecting actions greedily based on its current Q-values [23].

\section{Exploration in Multiobjective RL}

In this section we will consider how each of the exploration strategies discussed in 2.2 can be applied in the context of multiple objectives.

Extending $\epsilon$-greedy exploration to multiple objectives is straightforward. When an action is to be selected greedily, then the appropriate multi-objective action selection operation is used (TLO in our case). Otherwise an action is selected randomly. This has been the predominant exploration approach adopted in the MORL literature so far $[12,15,16,19,24,25,26,27,28,29$, $30,31,32,33,34,35,36,37,38,39,40,41,42,43,44,45]$.

Similarly optimistic initialisation can be applied quite easily to the multiobjective context. The only consideration is that as the range of values may vary between the different objectives, the optimal choice of initial optimistic value may also differ. Therefore a vector will be used to define the initial values, so that each objective may have its own optimistic initialisation.

Extending softmax to handle multiple objectives is more complicated, as Equation 4 can not be directly applied if $Q(s, a)$ is not a scalar. The appropriate approach to modifying softmax to handle vector $Q$ values depends on the nature of the multiobjective action selection method being used. If the action selection operation is based on a scalarisation operator which maps the vector value to a scalar, then the $Q$ values can simply be scalarised prior to applying the softmax equation. For example this approach could be used if the multiobjective action selection is being implemented via linear weighted scalarisation. This approach is shown in Equation 5, where $P(a)$ is the probability of selecting action $a, f$ is the scalarisation function, and $n$ is the number of actions.

$$
P(a)=\frac{e^{f(\bar{Q}(s, a)) / \tau}}{\sum_{b=1}^{n} e^{f(\bar{Q}(s, b)) / \tau}}
$$

However some approaches to multiobjective action selection can not readily be phrased as an explicit scalarisation operation. For example, the TLO 
approach can not be described by a simple scalarisation, and therefore Equation 5 can not be applied. Instead we need to define another means of converting the Q-value vectors into scalar values to which the softmax operator can be applied. This process should be consistent with the ordering of actions under the action selection operation - that is, if action $a$ is ranked higher than action $b$ by the non-linear action selection operator (i.e. if $U(\bar{Q}(s, a), \bar{Q}(s, b))>0$ ), it should be more likely to be selected than $b$ when the softmax operator is applied.

In this paper we propose two methods of achieving this aim. The first approach, which we will refer to as softmax-tournament or softmax-t, is based upon ranking the actions using the action-selection operator. Each action is assigned a score equal to the number of other actions which it is ranked above or equal with. These scores are then used as the basis for the softmax selection, as described in Algorithm 4.

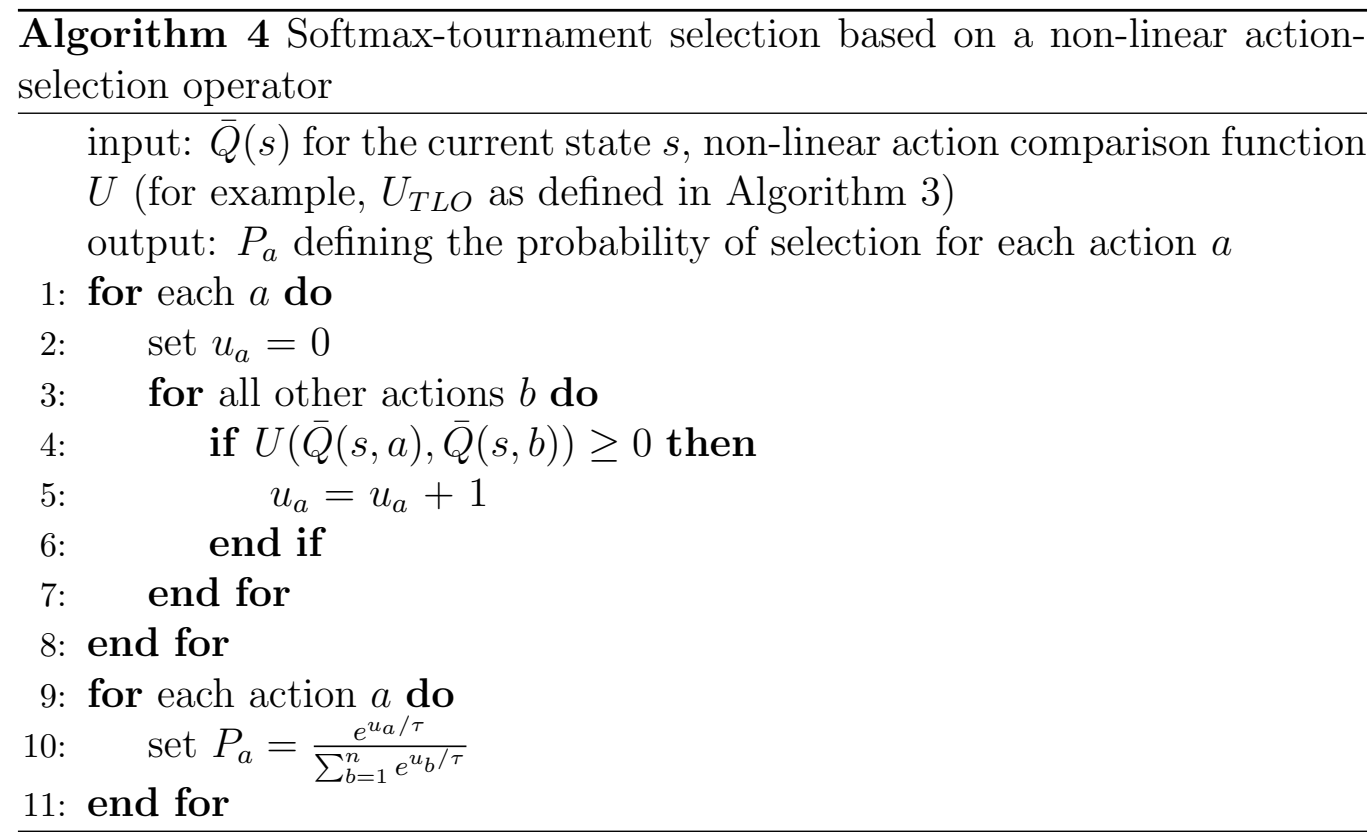

The softmax-t approach ensures that the probability with which an action will be selected is related to its ranking by the action selection operator in this sense it is consistent with the action selection operator. However the use of tournament scores may obscure the magnitude of the differences between actions. For example the highest ranked action will be selected 
with the same probability if it is only valued slightly higher than the secondranked action, or if it is valued substantially higher than the second-ranked action. The second approach we propose addresses this by basing the score of each action on the additive-epsilon metric $\left(\epsilon_{+}\right)$. This has been widely used in evolutionary multiobjective optimisation [46], and has previously been suggested as a metric for evaluating MORL algorithms by [47]. The additiveepsilon metric for an action is calculated as the maximum distance by which that action is valued below the greedy action on any individual objective, as shown in Figure 1. To make this value suitable for softmax selection, it needs to be inverted (so that a greater additive epsilon value results in a lower score). In addition the additive epsilon values should be calculated based on normalised objective values, to prevent an objective with a larger range from dominating these calculations. The overall softmax-epsilon (softmax-E) approach is summarised in Algorithm 5.

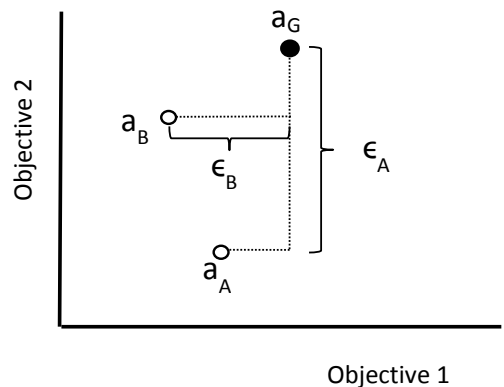

Figure 1: An illustration of the additive-epsilon metric. $a_{G}$ indicates the value of the greedy action with respect to each objective. $a_{A}$ and $a_{B}$ show the value of two other actions, and $\epsilon_{A}$ and $\epsilon_{B}$ indicate the additive-epsilon error metric for each of those actions.

\section{Experimental Methodology}

The remainder of this paper presents an empirical comparison of the multiobjective exploration strategies described in Section 3. To evaluate the effectiveness of each method in balancing the trade-off between exploration and exploitation it is necessary to consider the agent's performance both during learning (its online performance) and also the quality of the final greedy policy learnt by the agent (its offline performance). Ideally an agent should perform well on both of these measures. 


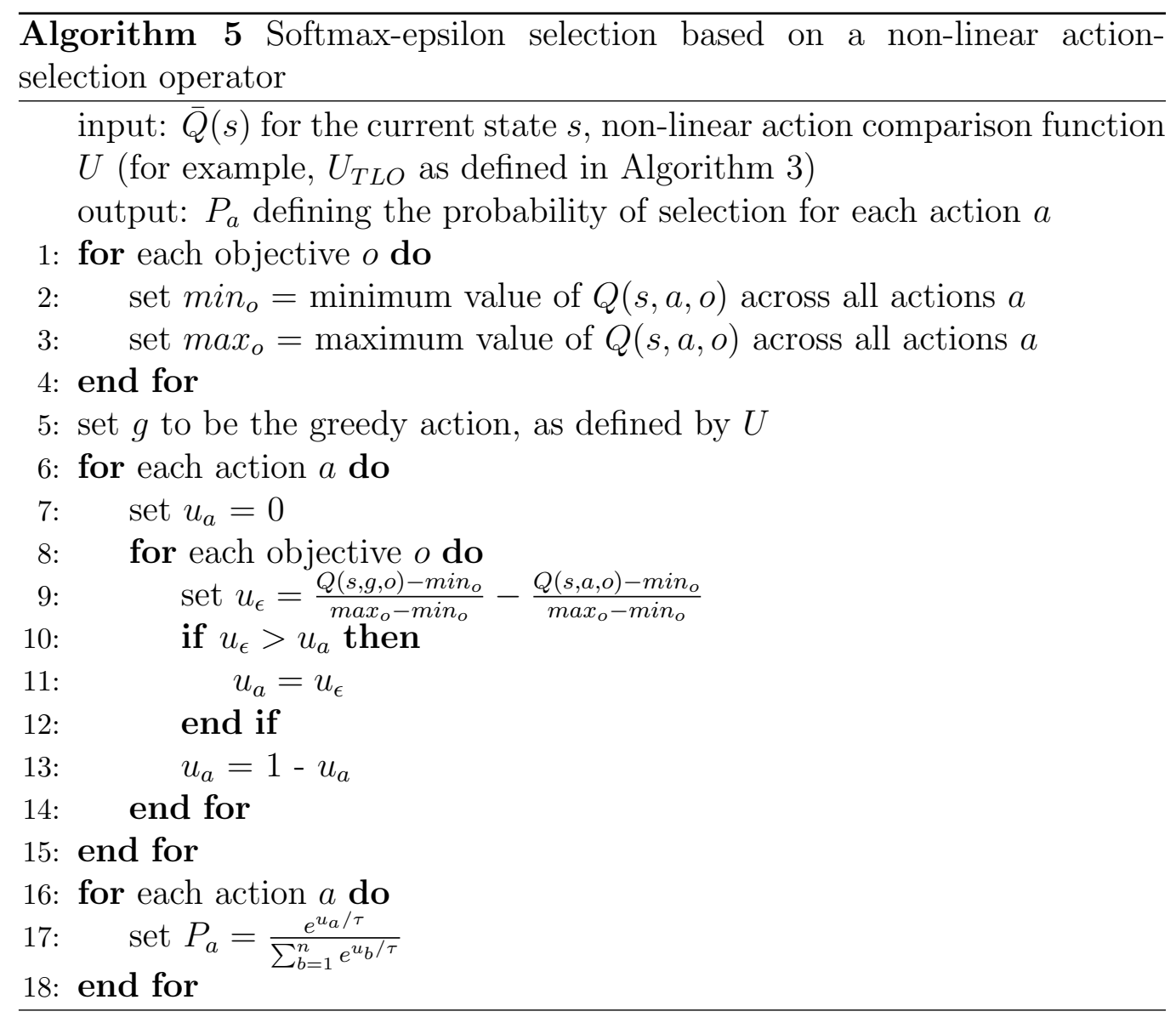


Evaluation of each strategy is complicated by the multiobjective nature of the rewards, which has two implications for experiments. First, it is necessary to evaluate each method's performance across a range of settings for the action-selection operation (in our case, the thresholds used by the TLO operation). This ensures that no bias is introduced by a method favouring a particular region of objective space. Secondly, comparison of methods is easier if the average vector rewards received during the offline and online phase can be represented as scalar values.

The approach previously proposed in the literature is to carry out several runs with different action-selection parameters, and then to summarise the set of vector results achieved via the hypervolume metric [39]. This approach is well suited if the actual Pareto front of policies for the test environment is not known. However in the case of the benchmark environments used in this study, the true values of the Pareto-optimal policies are known. Therefore we can more explicitly measure the ability of the agent to satisfy the desired criteria by comparing the results achieved by the agent against the optimal policy for the action-selection settings, as advocated by [47]. As illustrated in Figure 2, for each Pareto-optimal policy of a particular environment $\left(P_{A}, P_{B}\right.$ and $\left.P_{C}\right)$, a corresponding set of TLO threshold parameters can be determined which would result in the selection of that policy $\left(T_{A}, T_{B}\right.$ and $\left.T_{C}\right)$. For each of the threshold settings, the learning algorithm is executed and its performance on each objective is measured $\left(a_{A}, a_{B}\right.$ and $\left.a_{C}\right)$. The agent's mean reward is then compared to the expected per-episode reward for the corresponding Pareto-optimal policy. The vector difference between the expected and actual reward is then summarised as a scalar value using the additive-epsilon metric $(+\epsilon)$ as proposed by [47]. This process is carried out for both the agent's online and offline performance.

Note that as shown by points $a_{A}$ and $a_{B}$ in Figure 2, the agent's performance may lie either above or below the thresholds - however this does not affect the calculation of the additive-epsilon metric. It is also possible, as shown by point $a_{C}$, that the agent may in fact outperform the optimal policy on one of the objectives. This positive difference between the agent and the optimal policy is simply ignored when calculating the additive epsilon metric.

For each of the exploration methods, this process is repeated over 20 independent trials and further summarised as an online mean and offline mean over those trials. This is repeated for multiple parameter settings for each method, to investigate the sensitivity of each method to its parameters. For each benchmark a total of 5000 online (learning) episodes were executed 


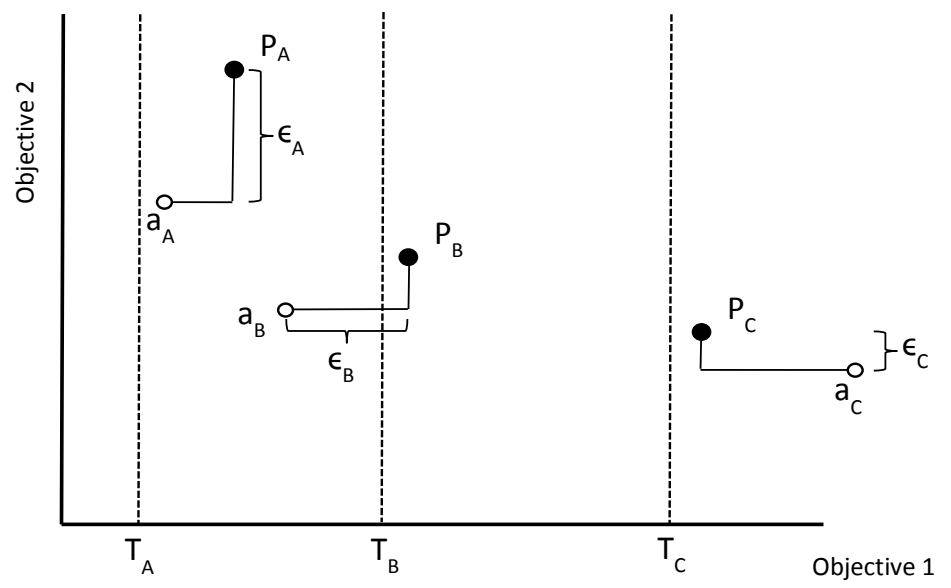

Figure 2: An illustration of the error measuring process used in the experiments. Dotted vertical lines indicate possible thresholds applied to the first objective, dark points indicate the corresponding Pareto-optimal policy for each threshold, and light points indicate the objective-space performance of an agent. The overall performance of this agent is the mean of the additive-epsilon error metrics $E_{A}, E_{B}$ and $E_{C}$.

in each trial, with one additional episode executed in strictly greedy fashion to evaluate the offline performance. The learning rate $\alpha$ was set to 0.9 as was the eligibility trace parameter $\lambda$ - these values were based on those utilised in prior research on the DST benchmark [19] and no attempt was made to tune these parameters for any of the individual methods. The exploration parameters were linearly decayed from their initial to final value over the online episodes - for $\epsilon$ the final value was 0 , while for $\tau$ the final value was $0.01^{1}$.

The experimental methodology is summarised below:

for each objective benchmark do

for 20 independent trials do

for each Pareto-optimal policy of that benchmark do

set the TLO parameters to the thresholds matching that policy

for 5000 learning episodes do

observe the reward vector received over the episode

calculate the $+\epsilon$ difference w.r.t the Pareto-optimal reward

\footnotetext{
${ }^{1}$ While not widely noted in the literature, numerical overflow errors can occur as the temperature $\tau$ nears zero. Limiting $\tau$ to a minimum value of 0.01 was sufficient to produce greedy behaviour by the end of training, while avoiding overflow errors.
} 
end for

calculate $+\epsilon$ for the final greedy policy (the offline performance) end for end for

report the mean online and offline $+\epsilon$ over all trials and thresholds

\section{end for}

In order for an empirical study to be of value the methods being investigated must be evaluated across a set of test environments, exhibiting the range of characteristics which might be encountered in practical application of the methods. This is difficult for MORL research due to the paucity of established benchmarks [39]. In addition, multiobjective Q-steering using TLO action-selection has been shown to suffer convergence problems when presented with environments in which more than one objective has non-zero rewards at non-terminal states [19]. Such problems are common to all TD algorithms using non-linear forms of action selection, due to the non-additive nature of the returns in this situation [5].

As the aim of this research is to evaluate the performance of the exploration methods rather than that of TLO or other non-linear approaches to action selection, it would be potentially misleading to include benchmarks with characteristics which inhibit TLO-based learning. Of the existing benchmarks in the literature, the widely-used Deep Sea Treasure problem is suitable for use in conjunction with TLO [39]. For this study we have introduced two further benchmark environments with characteristics which are suited to TLO. The details of each benchmark are given below.

\subsection{Deep Sea Treasure}

[39] proposes several benchmarks for empirical MORL, including the Deep Sea Treasure (DST) task (Figure 3). The DST has been widely adopted as a benchmark (e.g. $[15,32,43,44])$. The agent controls a submarine which starts from a location near the shore and travels out to sea to retrieve treasure. A reward of -1 is awarded for the time objective on each time-step, while the treasure reward is zero except when a treasure is encountered at which point it is equal to the value of that treasure (as shown in the grey cells in Figure 3). Therefore the agent must find a trade-off between the value of the treasure and the time taken to reach it. Under TLO this can be achieved by minimising the time penalty, subject to achieving a threshold value of treasure. The thresholds required to find each of the ten Paretooptimal policies are detailed in Table 1. This is an episodic task with no 
discounting. Episodes end when a treasure is recovered or after a maximum of 1000 time-steps.

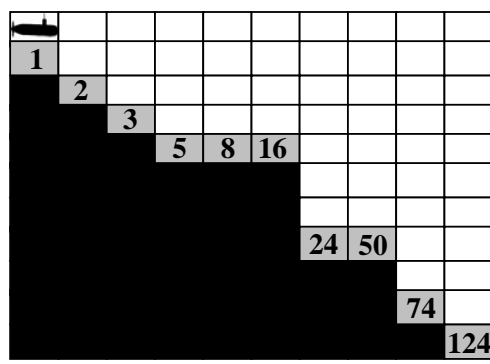

Figure 3: The Deep Sea Treasure task. Treasure values are indicated in the grey cells.

\begin{tabular}{ll}
\hline $\begin{array}{l}\text { Threshold } \\
\text { (for treasure objective) }\end{array}$ & $\begin{array}{l}\text { Optimal policy per-episode reward } \\
\text { (treasure, time) }\end{array}$ \\
\hline 0 & $(1,-1)$ \\
1.5 & $(2,-3)$ \\
2.5 & $(3,-5)$ \\
4 & $(5,-7)$ \\
6 & $(8,-8)$ \\
10 & $(16,-9)$ \\
20 & $(24,-13)$ \\
40 & $(50,-14)$ \\
60 & $(74,-17)$ \\
100 & $(124,-19)$ \\
\hline
\end{tabular}

Table 1: The set of Pareto-optimal policies and the corresponding TLO threshold parameter settings for the DST environment

\subsection{Bonus World}

Bonus World (Figure 4) is a new MORL benchmark introduced in this paper. Like DST it is a 2D episodic grid environment, but it has three objectives rather than two. Each episode starts with the agent in the location marked ' $S$ '. The agent can move in the four cardinal directions, and receives a reward of -1 for the time objective on every time-step. When reaching a terminal state (shown by grey cells) the agent receives the rewards specified in that cell for the other two objectives. In addition the rewards in the 
terminal states are doubled in magnitude if the agent has activated the bonus by visiting the cell marked 'X2'. The black cells near the bonus indicate walls which the agent can not pass through. Similarly the agent can not leave the bounds of the grid. Finally the cells marked 'PIT' indicate pits - if the agent enters one of these cells the bonus is deactivated, and the agent returns to the start state. A tabular representation of this environment has 162 discrete states -81 for the cells of the grid when the agent has not activated the bonus, and 81 for the same cells when the bonus has been activated. The set of Pareto-optimal policies and the corresponding thresholds are listed in Table 2. It can be seen that trade-offs exist between all three objectives. Note that not all optimal policies require the agent to activate the bonus.

\begin{tabular}{|c|c|c|c|c|c|c|c|c|}
\hline S & & & & & & & & $(1,9)$ \\
\hline & & & & & & & PIT & \\
\hline & & & & & & & & $(3,9)$ \\
\hline & & & X2 & & & & PIT & \\
\hline & & & & & & & & $(5,9)$ \\
\hline & & & & & & & PIT & \\
\hline & PIT & & PIT & & PIT & & & $(7,9)$ \\
\hline$(9,1)$ & & $(9,3)$ & & $(9,5)$ & & $(9,7)$ & & $(9,9)$ \\
\hline
\end{tabular}

Figure 4: The Bonus World task. Terminal reward values for objectives 1 and 2 are indicated in the grey cells, while a reward of -1 is received for the third objective (time) on each step. S marks the start location, X2 marks the location of the bonus, black cells indicate walls, and PIT marks the presence of a pit which returns the agent to the start location.

\subsection{Space Exploration}

The third benchmark is the Space Exploration task (Figure 5). The agent controls a spaceship which starts each episode in the location marked ' $S$ ' and aims to discover a habitable planet while minimising the amount of radiation to which it is exposed. A penalty of -1 is received for the radiation objective on all time-steps, except when in a region of high radiation (marked ' $R$ ') when the penalty is -11 . A positive reward is received for the mission success objective whenever a terminal state corresponding to a planet is reached - the magnitude of this reward reflects the desirability of that planet. If the ship enters a cell occupied by an asteroid, the ship is destroyed, the episode ends, and the agent receives a mission success reward of -100 . The threshold is applied to the mission success objective, meaning that the agent will attempt to minimise radiation exposure subject to meeting minimum habitability requirements. 


\begin{tabular}{ll}
\hline $\begin{array}{l}\text { Thresholds } \\
\text { (objective 1, objective 2) }\end{array}$ & $\begin{array}{l}\text { Optimal policy per-episode reward } \\
\text { (objective 1, objective 2, time) }\end{array}$ \\
\hline$(0,8)$ & $(1,9,-8)$ \\
$(2,8)$ & $(3,9,-10)$ \\
$(4,8)$ & $(5,9,-12)$ \\
$(10,12)$ & $(10,18,-14)$ \\
$(12,16)$ & $(14,18,-16)$ \\
$(16,16)$ & $(18,18,-18)$ \\
$(16,12)$ & $(18,14,-16)$ \\
$(12,10)$ & $(18,10,-14)$ \\
$(8,4)$ & $(9,5,-12)$ \\
$(8,2)$ & $(9,3,-10)$ \\
$(8,0)$ & $(9,1,-8)$ \\
\hline
\end{tabular}

Table 2: The set of Pareto-optimal policies and the corresponding TLO threshold parameter settings for the Bonus World environment

Unlike the other two environments, in Space Exploration the agent can move to all eight neighbouring states (i.e. there are eight actions). Also if the agent leaves the bounds of the grid, it moves to the opposite edge of the grid. For example if the agent moves up from the top row of the grid, it will move to the bottom row of the same column). This means that, unlike the other two benchmark environments, the execution of any action always results in a change in environmental state. The Pareto-optimal policies for this task and the corresponding thresholds are listed in Table 3.

\begin{tabular}{|l|l|l|l|l|l|l|l|l|l|l|l|l|}
\hline 20 & $\mathrm{R}$ & & & & & & & & 10 & $\mathrm{R}$ & $\mathrm{R}$ & 30 \\
\hline 20 & $\mathrm{R}$ & & & & & & & & 10 & $\mathrm{R}$ & $\mathrm{R}$ & 30 \\
\hline 20 & $\mathrm{R}$ & & & & $\mathrm{S}$ & & & & 10 & $\mathrm{R}$ & $\mathrm{R}$ & 30 \\
\hline 20 & $\mathrm{R}$ & & & & & & & & & $\mathrm{R}$ & $\mathrm{R}$ & 30 \\
\hline 20 & $\mathrm{R}$ & & & & & & & & & $\mathrm{R}$ & $\mathrm{R}$ & 30 \\
\hline
\end{tabular}

Figure 5: The Space Exploration task. S marks the start location, while grey cells represent the terminal states corresponding to planets, and black cells are terminal states corresponding to asteroids. Cells marked $\mathrm{R}$ have higher than normal levels of radiation.

\subsection{Comparison of Benchmarks}

The primary characteristic which differs between the three benchmark environments is the extent to which incorrect action choices can lead to states which are 'catastrophic' for the agent. The Space Exploration task 


\begin{tabular}{ll}
\hline $\begin{array}{l}\text { Threshold } \\
\text { for mission success objective }\end{array}$ & $\begin{array}{l}\text { Optimal policy per-episode reward } \\
\text { (mission success, radiation) }\end{array}$ \\
\hline 5 & $(10,-4)$ \\
15 & $(20,-15)$ \\
25 & $(30,-27)$ \\
\hline
\end{tabular}

Table 3: The set of Pareto-optimal policies and the corresponding TLO threshold parameter settings for the Space Exploration environment

is the most demanding with regards to this factor as the asteroid states result in immediate termination of the episode, and a reward which is very poor with respect to any of the Pareto-optimal policies. These states are also numerous and positioned within a single step of the optimal paths within the environment. The next most difficult environment is the DST, as terminal states exist which are close to the optimal path and which are sub-optimal with respect to most threshold settings - in particular the terminal state immediately below the starting state. The Bonus World problem is less challenging as each terminal state is generally at least two steps away from the optimal path for the other terminal states, and the pit locations which are within a single-step of the optimal path do not lead to episode termination, merely a penalty with regards to the time objective.

\section{Experimental Results}

\section{1. $\epsilon$-greedy exploration results}

$\epsilon$-greedy exploration is both the simplest form of exploration, and also the most widely-used in the MORL literature. Therefore the results achieved using this approach will be presented first as a baseline for comparison with the other approaches. Figure 6 illustrates both the online and offline error achieved when applying $\epsilon$-greedy exploration to each of the benchmarks with a range of different settings for the initial value of $\epsilon$. It can be seen that for the DST and Bonus World tasks this approach to exploration failed to find the optimal offline results on a consistent basis unless $\epsilon$ was set to a very high initial value. On the Space Exploration task, the offline performance was even poorer with no parameter setting being sufficient to guarantee finding the correct optimal policy.

The high initial values of $\epsilon$ required to optimise offline performance give rise to poorer online performance, as evident by the widening gap between 
the online and offline performance on the right-hand side of Figure 6. The initial setting of $\epsilon$ required to optimise online performance on each task is considerably lower than that required to optimise the offline performance. The issue here is that all problems contain terminal states which are not optimal with regards to the current TLO threshold settings. The random nature of the exploration produced by the $\epsilon$-greedy strategy is likely to encounter these states quite frequently. Entering one of these states results in a sub-optimal reward for the current episode. It also ends that episode, thereby limiting the agent's ability to learn from that episode.

\subsection{Softmax exploration results}

In contrast to the results observed for $\epsilon$-greedy exploration, both of the softmax exploration techniques were successfully able to learn the optimal offline policy for all three environments, as shown in Figures 7 and 8 . In general this was achieved at initial temperature settings corresponding to relatively low levels of exploration, therefore resulting in quite good online performance.

Direct comparison of the results in Figures 6 to 8 is difficult due to the differing parameters (and therefore different horizontal axes used in the figures). However as discussed earlier in the paper, and as is evident in these figures, the performance of each algorithm across much of its parameterspace can be seen as a trade-off between online and offline performance ${ }^{2}$. For any algorithm, the results achieved for a particular parameter setting can be treated as a point in a two-dimensional space, where one axis corresponds to online performance and the other to offline performance. By collating these points across all parameter settings for that algorithm and discarding any points which are Pareto-dominated, an approximate Pareto front can be produced. In this way the nature of the exploration-exploitation trade-off acheived by each algorithm can be visualised, as shown in Figure 9. By abstracting away from the parameters shown in Figures 6 to 8, these Pareto front graphs facilitate comparison of the performance of the different algorithms ${ }^{3}$.

\footnotetext{
${ }^{2}$ That is, exploration in RL is itself a multiobjective task.

${ }^{3}$ It should be noted that these figures, are not strictly Pareto fronts - both here, and in similar figures later in this paper, a small number of weakly dominated points have been included for purposes of clarity.
} 
It can be seen that both softmax methods clearly dominate $\epsilon$-greedy exploration on the DST and Space Exploration tasks. Both the DST and Space Exploration tasks exhibit 'failure' states located a single step away from the optimal path, which are more likely to be encountered via the random exploration of $\epsilon$-greedy than by the more directed softmax methods. In contrast the non-optimal terminal states in the Bonus World task are at least two steps away from the optimal path. This may explain why $\epsilon$-greedy and softmax-t have similar fronts on this task, although the latter is still slightly superior.

Softmax-E is clearly superior to softmax-t on the Bonus World and Space Exploration tasks, but slightly weaker on the Deep Sea Treasure environment.

\subsection{The effect of optimistic initialisation}

The results reported in Section 5.2 were obtained using the default initialisation of 0 for all objectives prior to the commencement of learning. We will refer to these results as those achieved using neutral initialisation, to distinguish them from the optimistic initialisation considered in this section ${ }^{4}$. The optimistic initial values used for all of the experiments reported in this section are listed in Table 4.

\begin{tabular}{ll}
\hline Environment & Initial values for each objective \\
\hline DST & $(150,0)$ \\
Bonus World & $(20,20,0)$ \\
Space Exploration & $(40,0)$ \\
\hline
\end{tabular}

Table 4: The initial value vectors used for each benchmark environment for all experiments involving optimistic initialisation.

In the single-objective RL literature, optimistic initialisation is often used in conjunction with greedy action selection [23]. That is, the optimistic nature of the initial values is the sole factor driving exploration. However the results shown in Table 5 indicate that this approach was extremely unsuccessful when applied in the context of Q-learning with multiple objectives and TLO action-selection, with both the online and offline performance being very poor.

\footnotetext{
${ }^{4}$ Note that 0 is not necessarily neutral for all objectives. For example on DST it is optimistic with respect to the time objective, and pessimistic with respect to the treasure objective. Truly neutral initialisation can only be achieved if the exact values of the optimal policy are known in advance, which will generally not be the case.
} 


\begin{tabular}{lll}
\hline Environment & Online mean & Offline mean \\
\hline DST & 578.2 & 579.0 \\
Bonus World & 306.4 & 306.5 \\
Space Exploration & 979.0 & 983.0 \\
\hline
\end{tabular}

Table 5: The mean online and offline results achieved using greedy exploration with optimistic initialisation on all three benchmarks.

A closer examination of the behaviour of the agent during these trials revealed that the cause of this performance was the agent becoming caught in loops of recurring states and actions. That is to say, once the agent reached a particular state $s$ it would execute a sequence of one or more actions which lead back to the same state $s$. The combination of optimistic initialisation, the reward structure of the environments, and the non-linear nature of the TLO action-selection could potentially result in that same sequence of actions continuing to be regarded as greedy by the agent regardless of how many times the sequence has been executed.

This is best illustrated by an example. Consider the case where the agent is currently occupying a state in the upper row of the DST, and the threshold for the treasure objective has been set at 100. Assume that all actions other than moving up have previously been explored and eventually lead to terminal states, so their Q-values have been trained downwards (e.g. to $(50,-6)$ ) while the Q-values for the up action remain at their initial setting of $(150,0)$. Clearly up will be regarded as the greedy action, and if executed will result in a reward of $(0,-1)$ and the agent remaining in the same state. As the agent remains in the same state, no discounting is being applied, and the immediate reward for the treasure objective is zero, the estimated Q-value for this state-action pair for the treasure objective will remain unchanged. In contrast the immediate reward of -1 for the time objective will result in the Q-value for that objective being lowered. Assuming $\alpha$ of 0.9 , the updated Q-value for this action will now be $(150,-0.9)$. As the estimated treasure value remains above the threshold, this action will continue to be regarded as the greedy action. No matter how many times the action is executed it will remain the greedy choice, as the Q-value for the treasure objective is never trained downwards. The estimated value of the time objective for this action will steadily train downwards - however in this situation TLO does not take this objective into account as this is the only action exceeding the threshold value for the first objective. Therefore this action will be repeatedly 
executed until the agent chooses an exploratory action. This leads to poor online performance, and also can result in the agent failing to find the optimal offline policy. While the example given here consists of a single action, recurrent sequences of two or more actions were also observed. In particular the structure of the Space Exploration task is such that a single action can not leave the environment's state unchanged, but recurrent behaviour was still evident in the results on this task.

The results in Table 5 clearly show that the combination of optimistic initialisation and greedy action-selection is not adequate for the tasks examined in this paper. However, optimistic initialisation may still be beneficial when combined with some other form of exploration. Figures 10 to 12 compare the performance of each exploration method under neutral and optimistic initialisation. It can be seen that the incorporation of even a small amount of additional exploration in conjunction with optimistic initialisation is sufficient to overcome the problem highlighted in Table 5, and allow learning of the optimal offline policy.

When the performance of each algorithm with neutral initialisation is compared against the same algorithm using optimistic initialisation (Figures 10 to 12 ), it can be seen that the optimistically initialised agent is generally able to learn the optimal offline policy at much lower settings for the exploration parameter $(\epsilon$ or $\tau)$, which in turn leads to marked improvements in the online performance. The only exception is for the combination of softmax exploration with very low initial values of $\tau$ where the optimistically initialised agent still exhibits signs of the problematic behaviour observed for greedy action-selection. Softmax-E performed more poorly than softmax-t under these conditions as reflected by the different vertical scales required for the DST and Space Exploration graphs in Figures 11 and 12. The reason is that under optimistic initialisation very large differences in Q-values can easily arise early in learning. For example in the Space Exploration task, if most actions from the current state have been found to lead to asteroids, then any untried actions will be valued much more highly than those actions which have been experienced. By focusing only on the rankings of actions rather than the magnitude of the differences, softmax-t somewhat reduces the impact of these early variations in values when compared to softmax-E. 

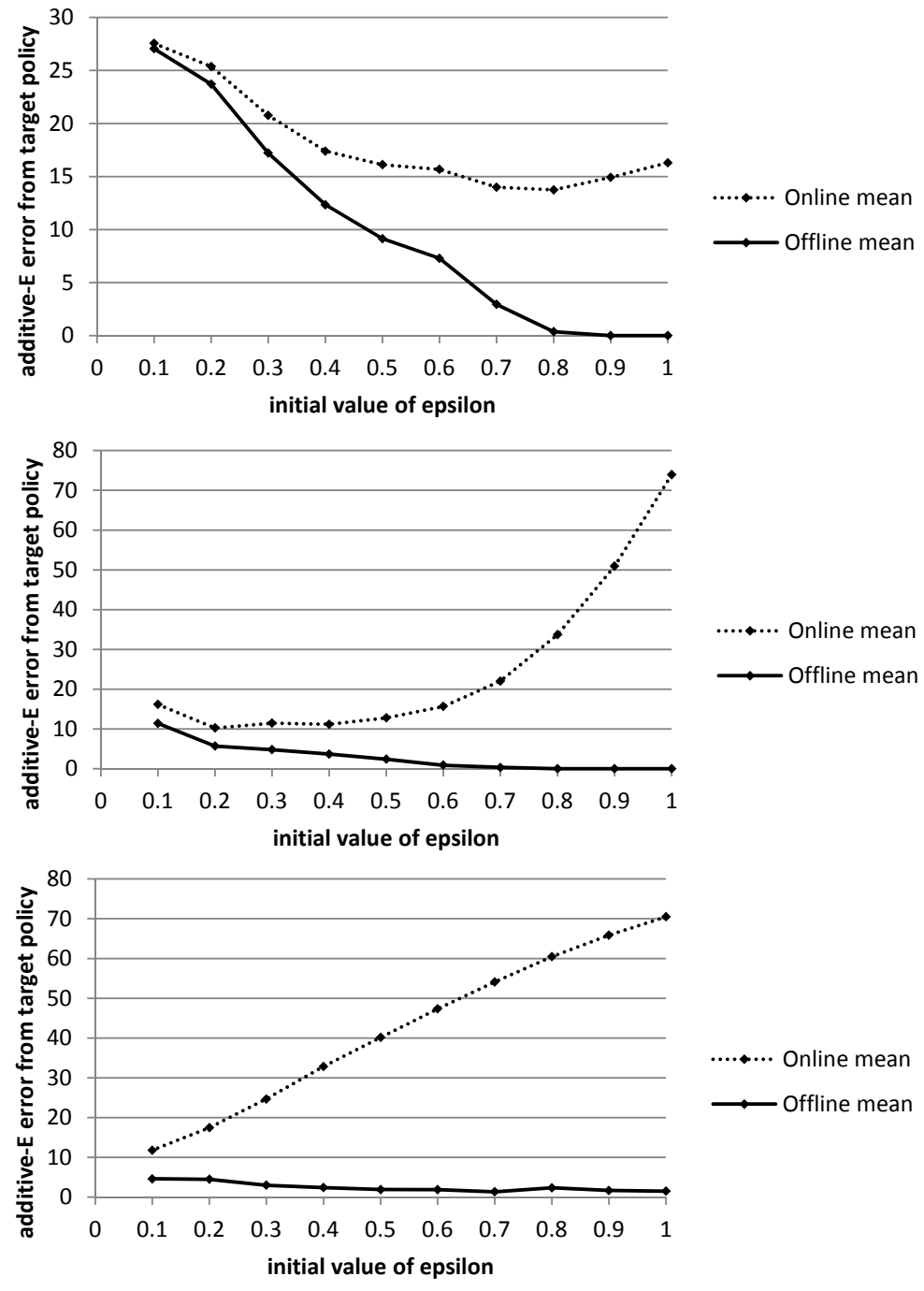

Figure 6: The mean online and offline error achieved using $\epsilon$-greedy exploration on the Deep Sea Treasure (top), Bonus World (middle), and Space Exploration (bottom) environments. 

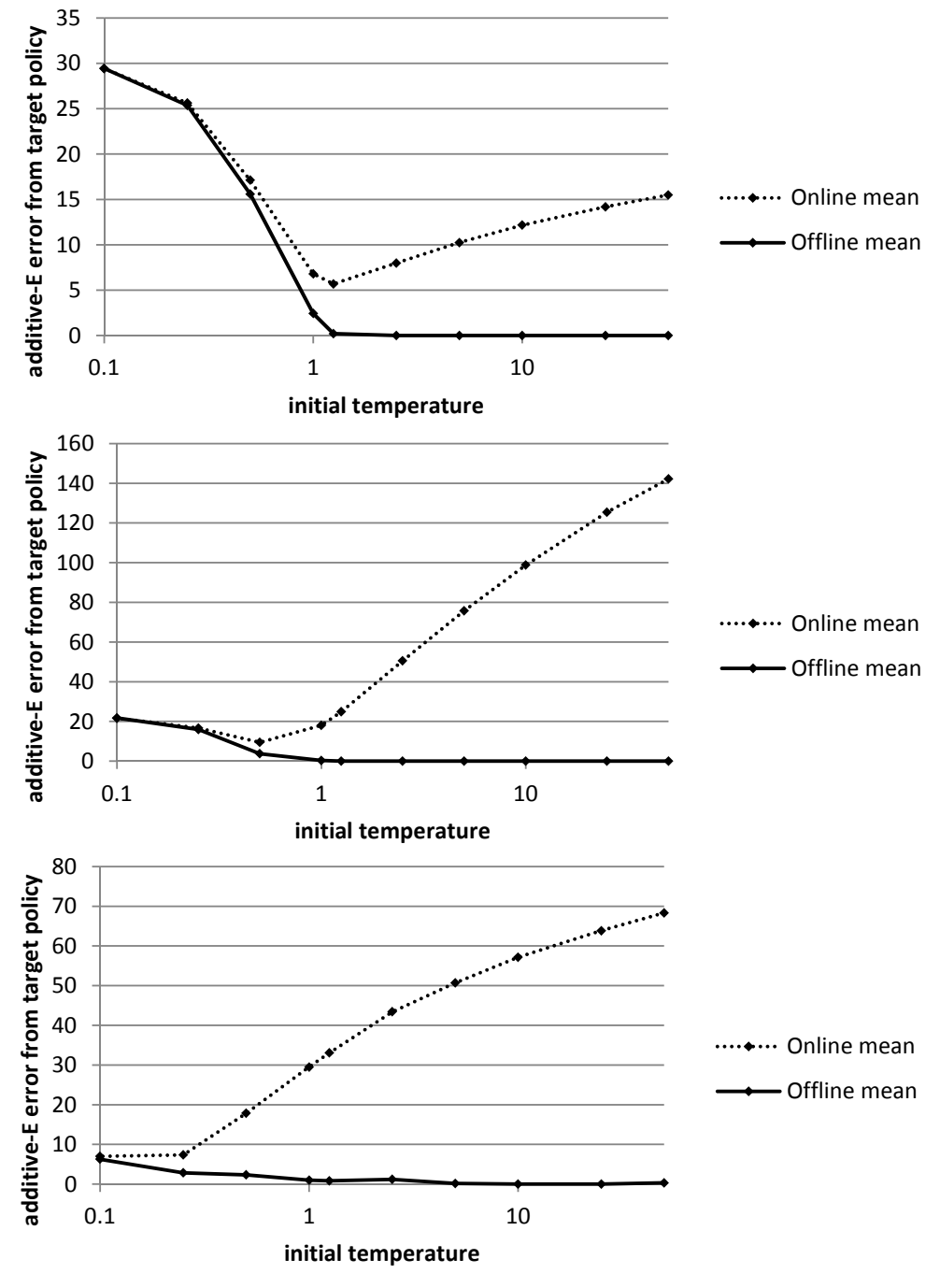

Figure 7: The mean online and offline error achieved using softmax-t exploration on the Deep Sea Treasure (top), Bonus World (middle), and Space Exploration (bottom) environments. 

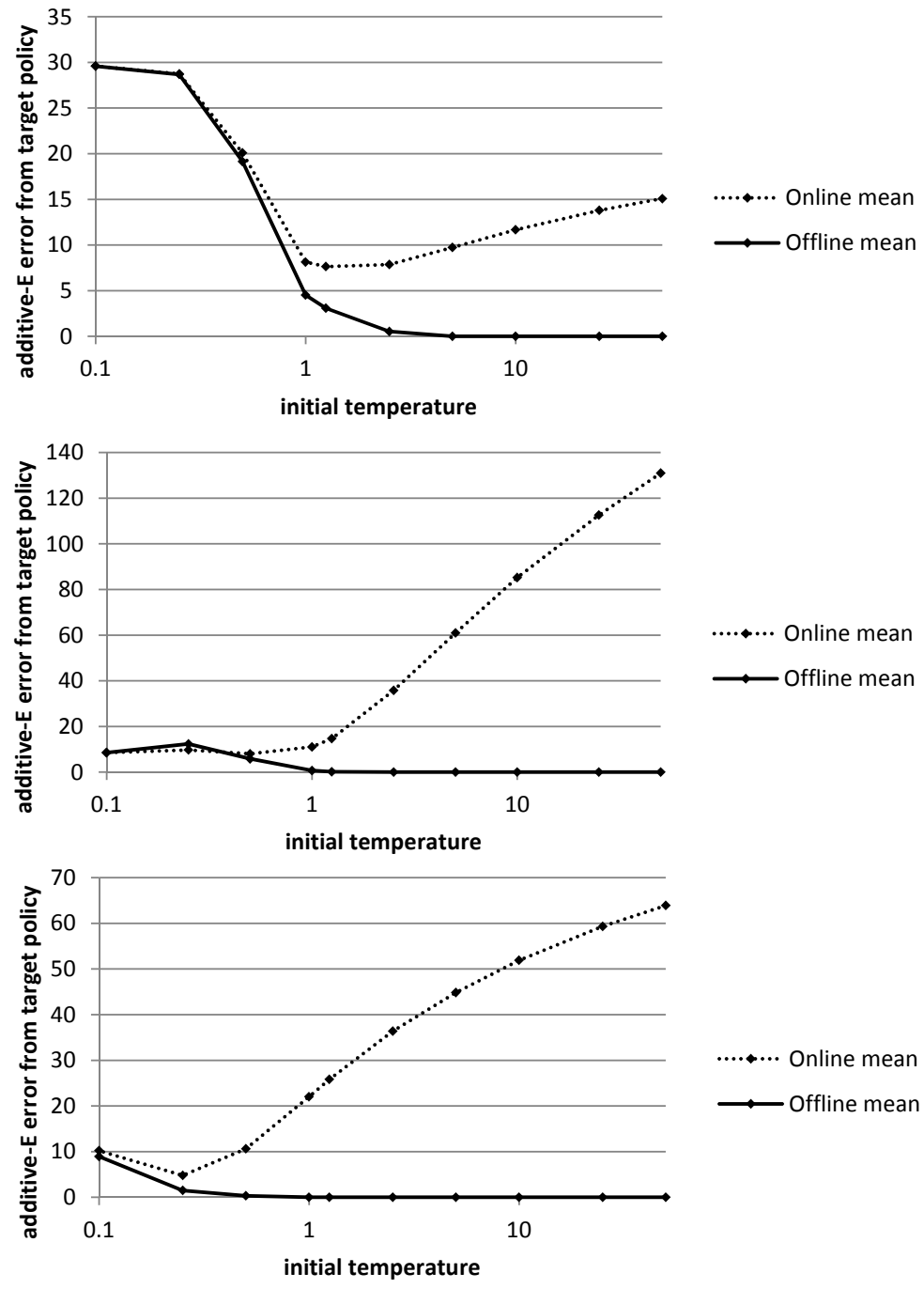

Figure 8: The mean online and offline error achieved using softmax-E exploration on the Deep Sea Treasure (top), Bonus World (middle), and Space Exploration (bottom) environments. 

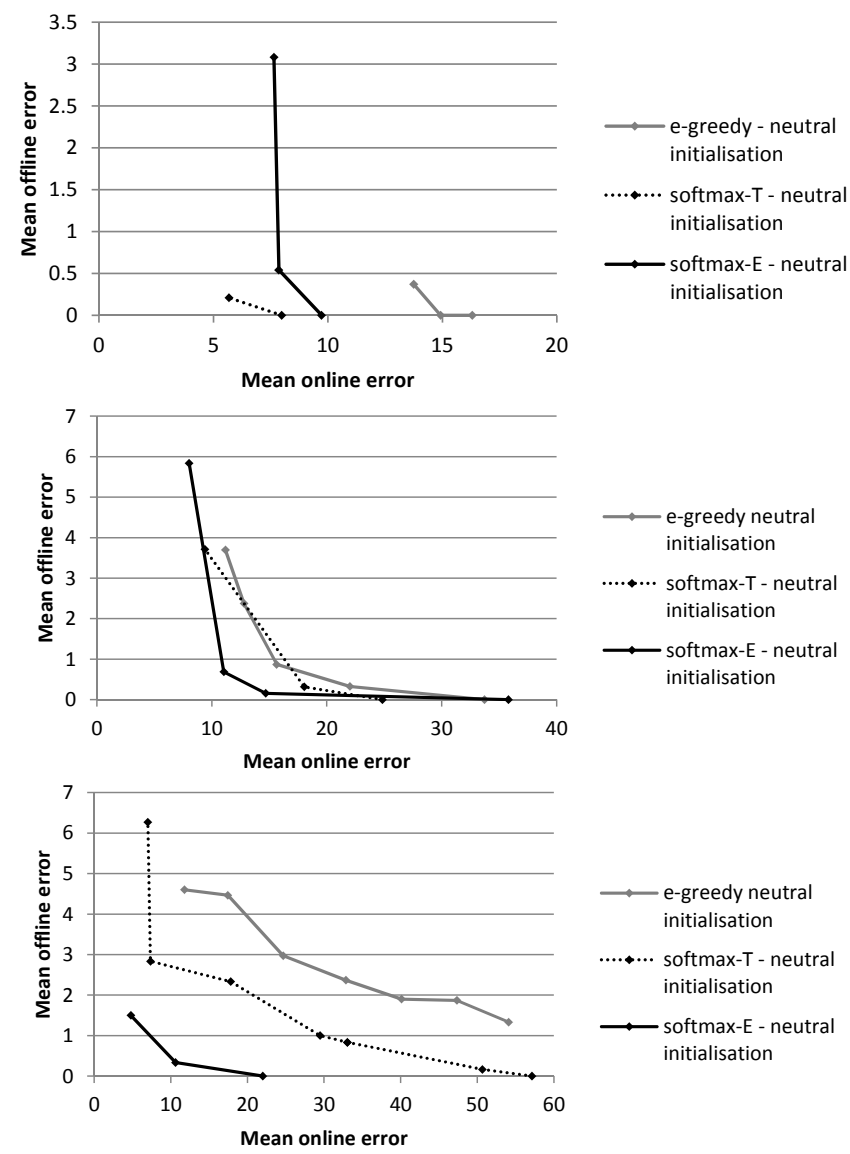

Figure 9: Pareto-front visualisation of the tradeoff between mean online and offline error achieved using $\epsilon$-greedy exploration and both variants of softmax exploration on the Deep Sea Treasure (top), Bonus World (middle), and Space Exploration (bottom) environments. 

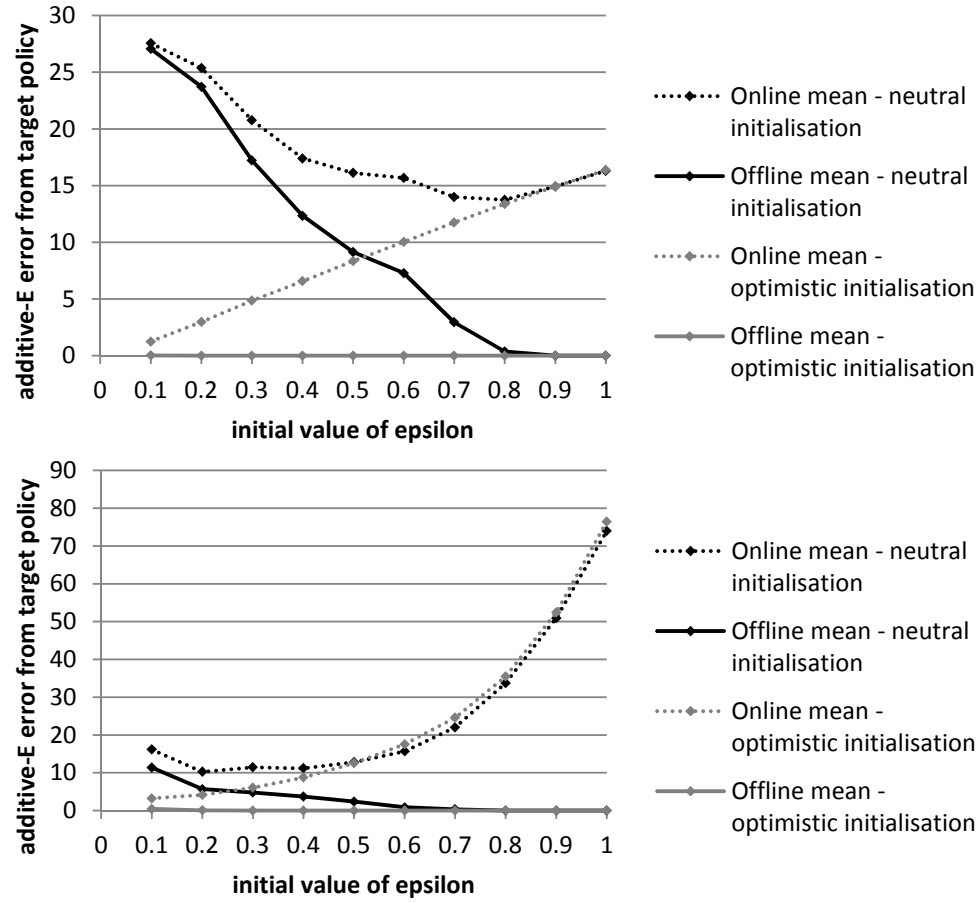

....... Online mean - neutral initialisation

$\longrightarrow$ Offline mean - neutral initialisation

....... Online mean optimistic initialisation

$\longrightarrow$ Offline mean optimistic initialisation

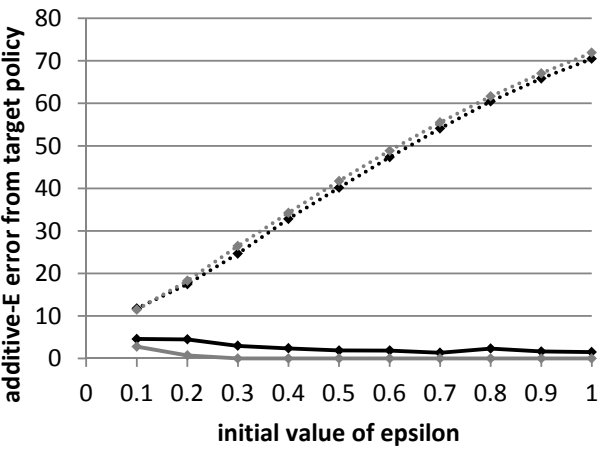

....... Online mean - neutral initialisation

$\longrightarrow$ Offline mean - neutral initialisation

....... Online mean optimistic initialisation

Offline mean optimistic initialisation

Figure 10: The mean online and offline error achieved using $\epsilon$-greedy exploration with both neutral and optimistic initialisation on the Deep Sea Treasure (top), Bonus World (middle), and Space Exploration (bottom) environments. 

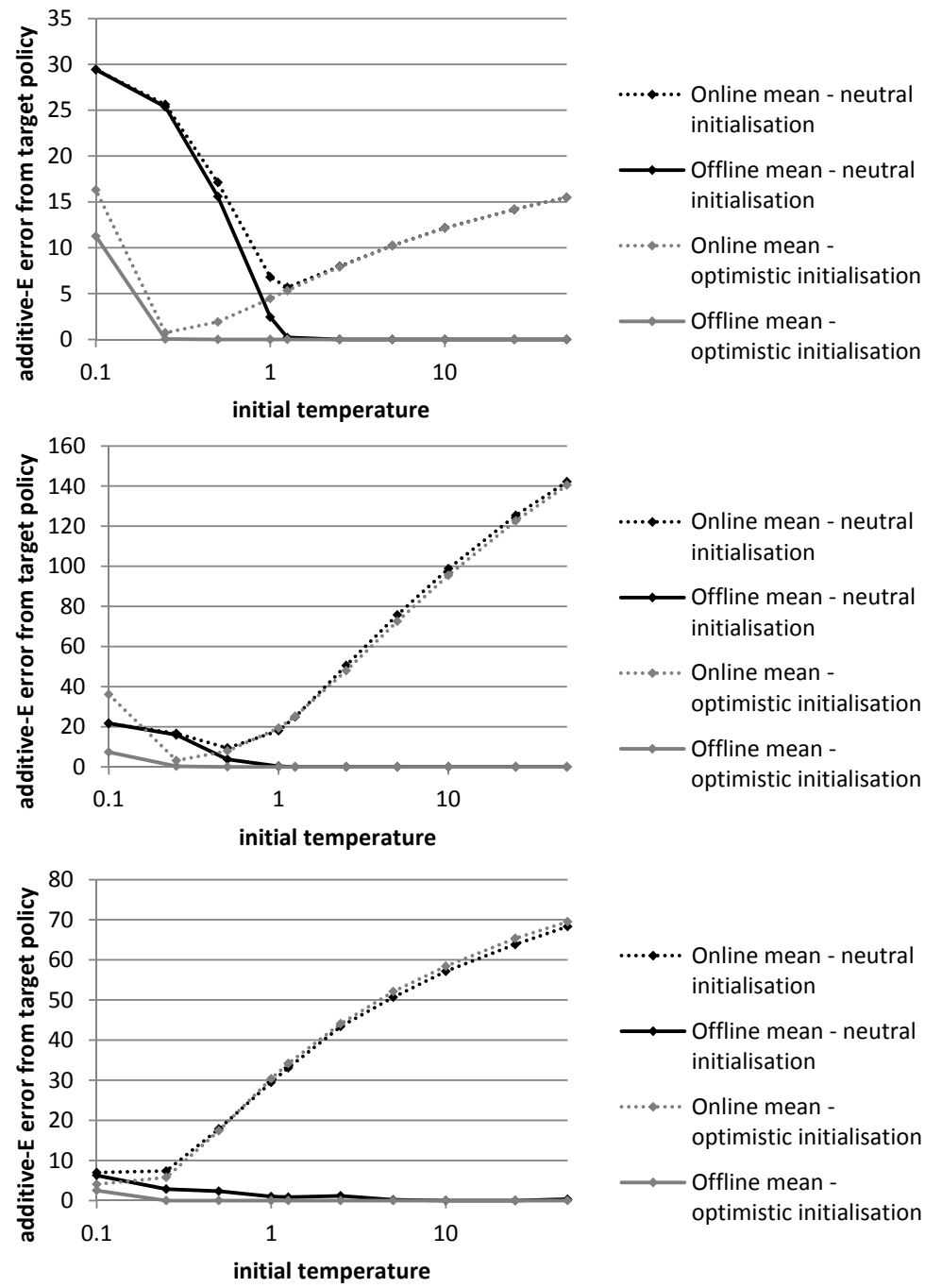

....... Online mean - neutral initialisation

$\longrightarrow$ Offline mean - neutral initialisation

....... Online mean optimistic initialisation

$\longrightarrow$ Offline mean optimistic initialisation

Figure 11: The mean online and offline error achieved using softmax-t exploration with both neutral and optimistic initialisation on the Deep Sea Treasure (top), Bonus World (middle), and Space Exploration (bottom) environments. 

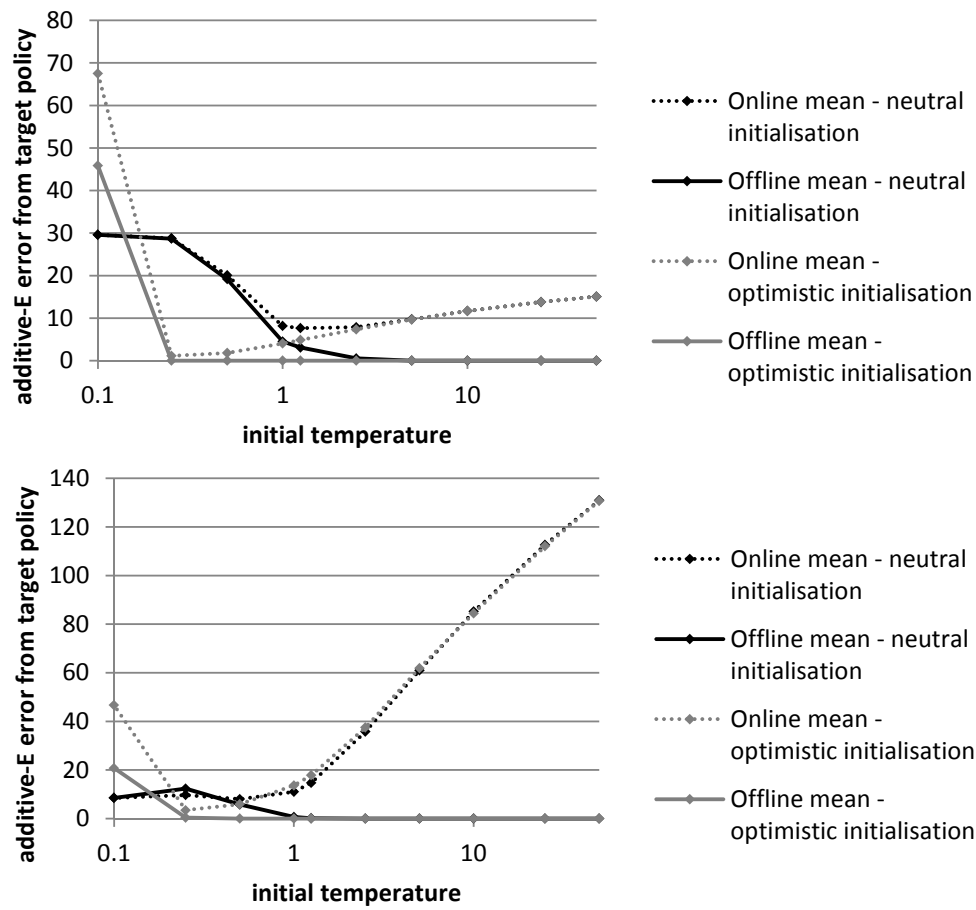

....... Online mean - neutral initialisation

$\longrightarrow$ Offline mean - neutral initialisation

........ Online mean optimistic initialisation

$\because$ Offline mean optimistic initialisation
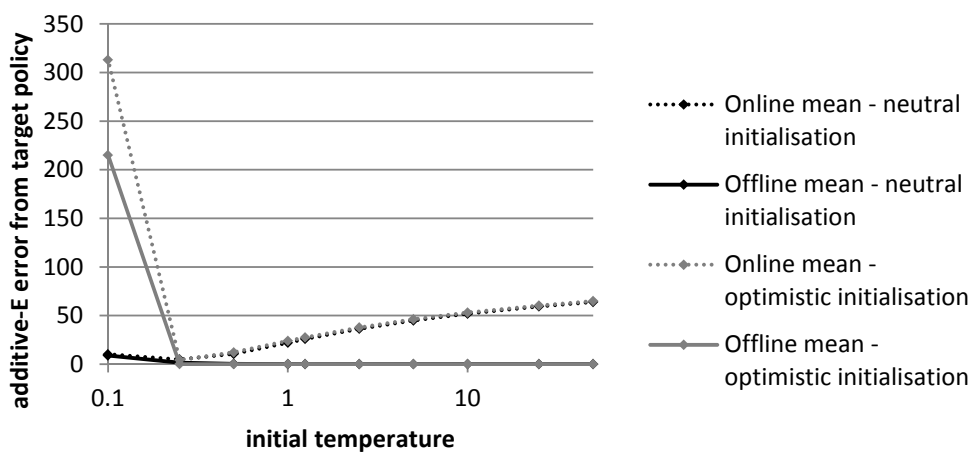

Figure 12: The mean online and offline error achieved using softmax-E exploration with both neutral and optimistic initialisation on the Deep Sea Treasure (top), Bonus World (middle), and Space Exploration (bottom) environments. 
The impact of optimistic initialisation is further illustrated by the Pareto front visualisations in Figures 13 to 15. It can be seen that for every exploration strategy and every environment, the Pareto front resulting from optimistic initialisation clearly dominates that produced using neutral initialisation.
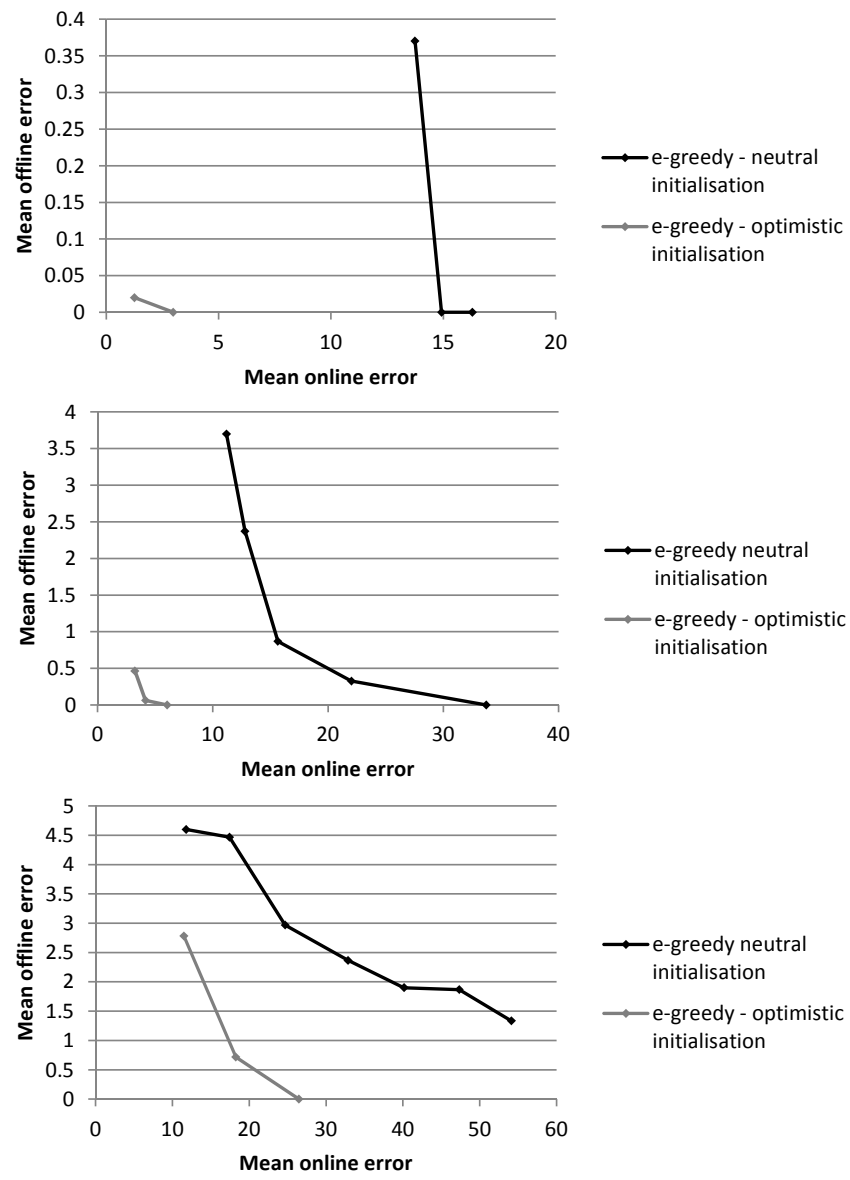

Figure 13: Pareto-front visualisation of the tradeoff between mean online and offline error achieved using $\epsilon$-greedy exploration with both neutral and optimistic initialisation on the Deep Sea Treasure (top), Bonus World (middle), and Space Exploration (bottom) environments. 

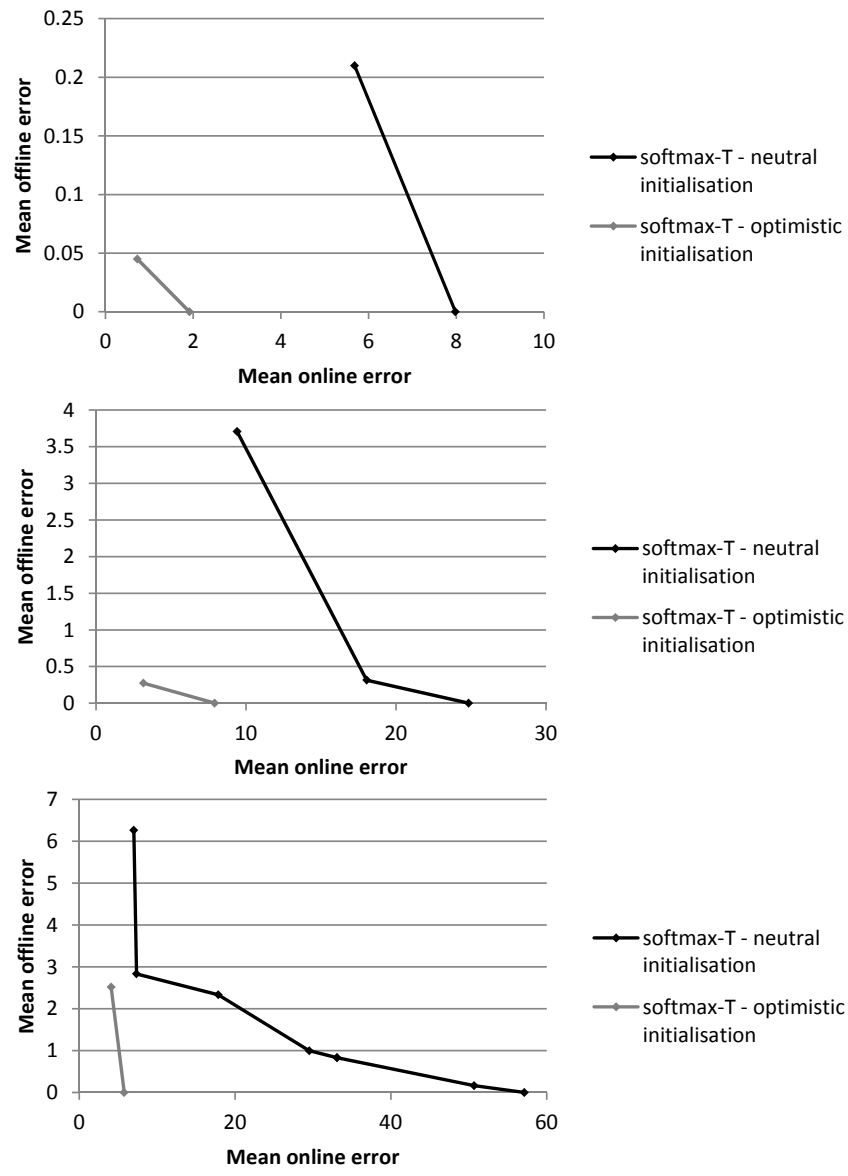

Figure 14: Pareto-front visualisation of the tradeoff between mean online and offline error achieved using softmax-t exploration with both neutral and optimistic initialisation on the Deep Sea Treasure (top), Bonus World (middle), and Space Exploration (bottom) environments. 

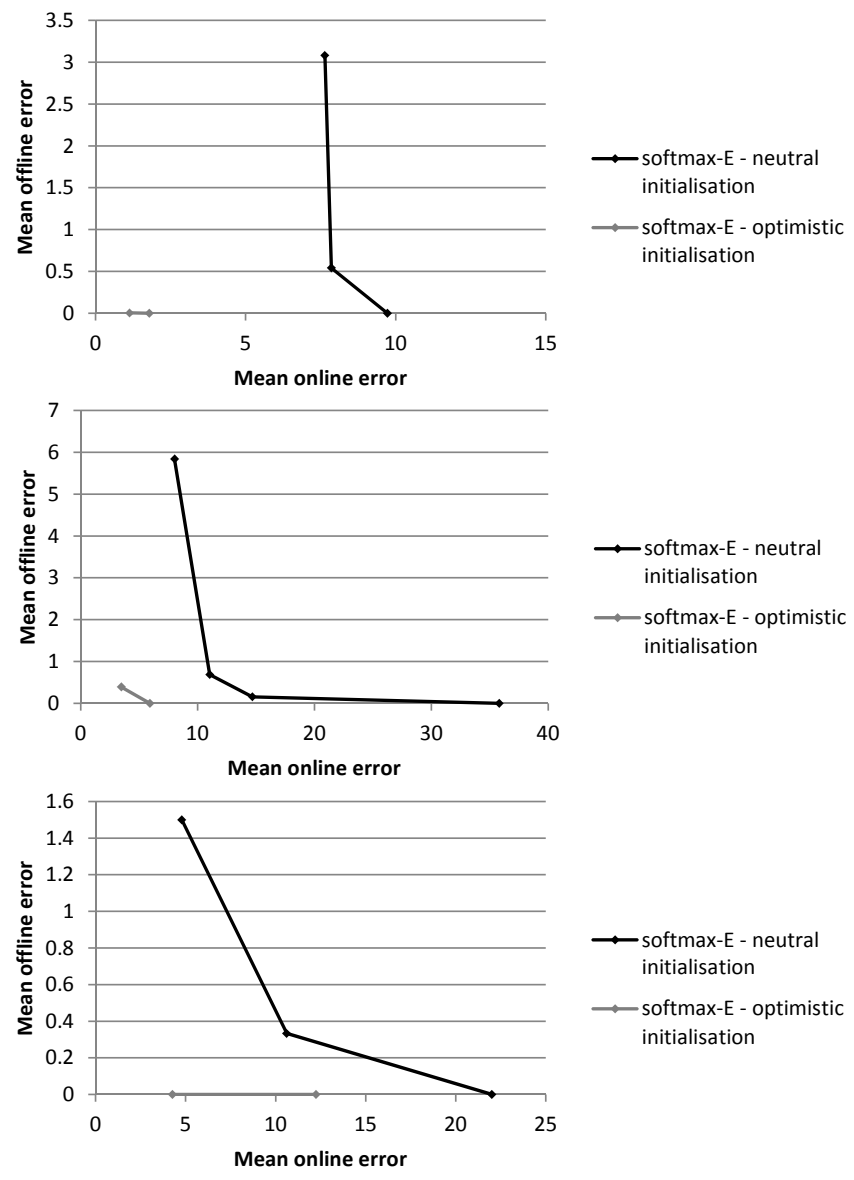

Figure 15: Pareto-front visualisation of the tradeoff between mean online and offline error achieved using softmax-E exploration with both neutral and optimistic initialisation on the Deep Sea Treasure (top), Bonus World (middle), and Space Exploration (bottom) environments. 

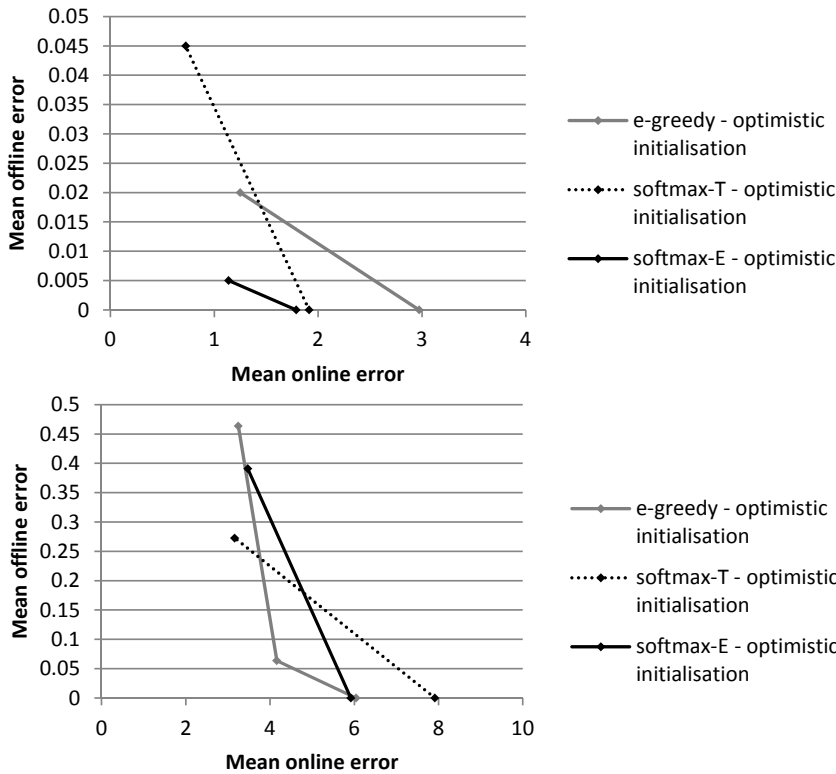

$\longrightarrow$ e-greedy - optimistic initialisation

....... softmax-T - optimistic initialisation

$\longrightarrow$ softmax-E - optimistic initialisation

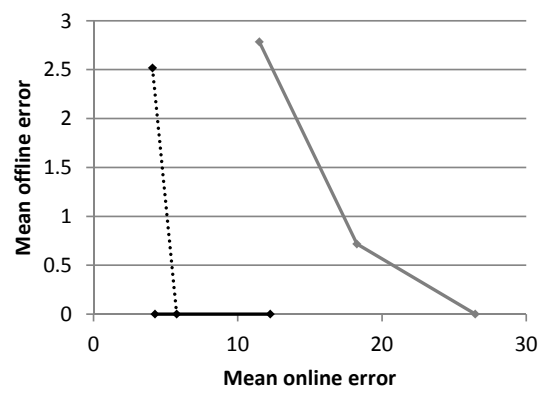

e-greedy - optimistic initialisation

....... softmax-T - optimistic initialisation

softmax-E - optimistic initialisation

Figure 16: Pareto-front visualisation of the tradeoff between mean online and offline error achieved using $\epsilon$-greedy exploration and both variants of softmax exploration with optimistic initialisation on the Deep Sea Treasure (top), Bonus World (middle), and Space Exploration (bottom) environments.

Figure 16 provides a comparison of the overall performance of $\epsilon$-greedy, softmax-t and softmax-E exploration across the three benchmark problems, using optimistic initialisation. On both the DST and Space Exploration environments both softmax methods clearly outperform $\epsilon$-greedy exploration. Even though the performance of $\epsilon$-greedy on these problems is improved by optimistic initialisation, the random exploration still encounters the 'failure' states more frequently than for the softmax methods. The results on the Bonus World task are less clear-cut, with the $\epsilon$-greedy and softmax-E fronts having similar end-points - this environment lacks the catastrophic failure 
states which impede $\epsilon$-greedy's performance on the other environments. Over all three environments, softmax-E appears to perform slightly better than softmax-t.

\section{Conclusion and Future Work}

This work has established several benchmarks for evaluating exploration in MORL, and has used these to highlight issues which may arise when applying the simple exploration methods commonly used in single-objective RL in a multiobjective context. In particular it has been shown that the simple combination of optimistic initialisation and greedy action-selection which has been widely used in single-objective RL performs extremely poorly when applied to multiobjective problems in combination with the TLO approach to action-selection. The combination of the environmental reward structure and TLO can lead to the agent repeatedly executing recurrent series of action(s) and therefore failing to learn the optimal policy. However the combination of optimistic initialisation with other forms of exploration can be very effective.

We have shown that the softmax approach to exploration can be extended to handle multiple objectives. Of the two variants examined in this work, the softmax-E method (which combines softmax exploration with the additiveepsilon metric) appears to provide the most effective trade-off between exploration and exploitation. In particular this approach in combination with optimistic initialisation produced strong online and offline performance across all three benchmark environments. While the results reported here are specific to TLO action-selection, the softmax-E approach would be equally applicable in combination with any non-linear form of action-selection.

Future work needs to examine whether more sophisticated approaches to exploration can be adapted or developed for multiobjective RL. One promising direction is the Exploration Objective Value Function (EOVF) approach recently proposed by [48]. This approach essentially 'multiobjectifies' a single-objective problem by adding an additional, optimistically initialised 'novelty' objective. This receives a reward of zero on all time-steps and is discounted so that its values eventually decay to zero. The separation of optimistic initialisation into a separate objective may address some of the issues observed in this paper. However [48] use a linear weighted combination of objectives, so it remains to be seen how EOVF will function in the context of non-linear action selection. 
An additional line of further investigation will be to examine how well various exploration methods operate for multi-policy MORL algorithms such as the Pareto set approach of [37]. In order to find a wide range of Paretooptimal policies, multi-policy methods are likely to require different forms or levels of exploration than single-policy approaches, but this issue has yet to be properly investigated.

[1] Y. Zhang, Y. Jun, G. Wei, L. Wu, Find multi-objective paths in stochastic networks via chaotic immune PSO, Expert Systems with Applications 37 (2010) 1911-1919.

[2] S. Wang, J. Yang, G. Liu, S. Du, J. Yan, Multi-objective path finding in stochastic networks using a biogeography-based optimization method, SIMULATION (2016) 637-647.

[3] S. Wen, B. Hu, H. Lam, Reinforcement learning optimization for base station sleeping strategy in coordinated multipoint (CoMP) communications, Neurocomputing 167 (2015) 443-450.

[4] Q. Wei, H. Zhang, J. Dai, Model-free multiobjective approximate dynamic programming for discrete-time nonlinear systems with general performance index functions, Neurocomputing 72 (2009) 1839-1848.

[5] D. Roijers, P. Vamplew, S. Whiteson, R. Dazeley, A survey of multiobjective sequential decision-making, Journal of Artificial Intelligence Research 48 (2013) 67-113.

[6] S. Thrun, Efficient Exploration In Reinforcement Learning, Technical Report CMU-CS-92-102, Carnegie-Mellon University, 1992.

[7] M. Drugan, B. Manderick, Exploration versus exploitation trade-off in infinite horizon pareto multi-armed bandits algorithms, in: The Seventh Conference of Agents and Artificial Intelligence, 2015.

[8] M. Drugan, A. Nowe, Designing multi-objective multi-armed bandits algorithms: A study, in: International Joint Conference on Neural Networks, IEEE, 2013, pp. 1-8.

[9] P. C. Fishburn, Utility theory for decision making, Technical Report, DTIC Document, 1970. 
[10] C. Liu, X. Xu, D. Hu, Multiobjective reinforcement learning: A comprehensive overview, Systems, Man, and Cybernetics: Systems, IEEE Transactions on 45 (2015) 385-398.

[11] Y. Guo, A. Zeman, R. Li, A reinforcement learning approach to setting multi-objective goals for energy demand management, International Journal of Agent Technologies and Systems 1 (2009) 55-70.

[12] J. Perez, C. Germain-Renaud, B. Kegl, C. Loomis, Responsive elastic computing, in: International Conference on Autonomic Computing, 2009, pp. 55-64.

[13] P. Vamplew, J. Yearwood, R. Dazeley, A. Berry, On the limitations of scalarisation for multi-objective reinforcement learning of Pareto fronts, in: AI'08: The 21st Australasian Joint Conference on Artificial Intelligence, Springer, 2008, pp. 372-378.

[14] Z. Gabor, Z. Kalmar, C. Szepesvari, Multi-criteria reinforcement learning, in: The Fifteenth International Conference on Machine Learning, 1998, pp. 197-205.

[15] K. Van Moffaert, M. Drugan, A. Nowé, Hypervolume-based multiobjective reinforcement learning, in: Evolutionary Multi-Criterion Optimization, 2013, pp. 352-366.

[16] K. Van Moffaert, M. M. Drugan, A. Nowé, Scalarized multi-objective reinforcement learning: Novel design techniques, in: Proceedings of the IEEE Symposium on Adaptive Dynamic Programming and Reinforcement Learning. IEEE, 2013, pp. 191-199.

[17] P. Geibel, Reinforcement learning for MDPs with constraints, in: European Conference on Machine Learning, volume 4212, 2006, pp. 646-653.

[18] P. Geibel, F. Wysotzki, Risk-sensitive reinforcement learning applied to control under constraints, Journal of Artificial Intelligence Research (2005) 81-108.

[19] R. Issabekov, P. Vamplew, An empirical comparison of two common multiobjective reinforcement learning algorithms, in: AI2012: The 25th Australasian Joint Conference on Artificial Intelligence, 2012, pp. 626636. 
[20] M. A. Wiering, Explorations in efficient reinforcement learning (1999).

[21] S. Ishii, W. Yoshida, J. Yoshimoto, Control of exploitation-exploration meta-parameter in reinforcement learning, Neural networks 15 (2002) 665-687.

[22] J. Asmuth, L. Li, M. L. Littman, A. Nouri, D. Wingate, A bayesian sampling approach to exploration in reinforcement learning, in: Proceedings of the Twenty-Fifth Conference on Uncertainty in Artificial Intelligence, AUAI Press, 2009, pp. 19-26.

[23] M. Grześ, D. Kudenko, Improving optimistic exploration in model-free reinforcement learning, Adaptive and Natural Computing Algorithms, Springer, 2009, pp. 360-369.

[24] T. Brys, A. Nowé, D. Kudenko, M. E. Taylor, Combining multiple correlated reward and shaping signals by measuring confidence, in: TwentyEighth AAAI Conference on Artificial Intelligence (AAAI-14), 2014.

[25] T. Brys, T. T. Pham, M. E. Taylor, Distributed learning and multiobjectivity in traffic light control, Connection Science 26 (2014) 65-83.

[26] L. A. Ferreira, R. A. Bianchi, C. H. Ribeiro, Multi-agent multiobjective learning using heuristically accelerated reinforcement learning, in: Robotics Symposium and Latin American Robotics Symposium (SBR-LARS), 2012 Brazilian, IEEE, 2012, pp. 14-20.

[27] L. A. Ferreira, C. H. C. Ribeiro, R. A. da Costa Bianchi, Heuristically accelerated reinforcement learning modularization for multi-agent multiobjective problems, Applied Intelligence 41 (2014) 551-562.

[28] C. Germain-Renaud, J. Perez, B. Kégl, C. Loomis, Grid differentiated services: a reinforcement learning approach, in: 8th IEEE International Symposium on Cluster Computing and the Grid, 2008.

[29] H. Iima, Y. Kuroe, Multi-objective reinforcement learning for acquiring all pareto optimal policies simultaneously-method of determining scalarization weights, in: Systems, Man and Cybernetics (SMC), 2014 IEEE International Conference on, IEEE, 2014, pp. 876-881. 
[30] N. Mehta, S. Natarajan, P. Tadepalli, A. Fern, Transfer in variablereward hierarchical reinforcement learning, Machine Learning 73 (2008) 289-312.

[31] A. Moustafa, M. Zhang, Multi-objective service composition using reinforcement learning, in: Service-Oriented Computing, Springer, 2013, pp. $298-312$.

[32] Y. Mukai, Y. Kuroe, H. Iima, Multi-objective reinforcement learning method for acquiring all Pareto optimal policies simultaneously, in: IEEE International Conference on Systems, Man and Cybernetics, 2012, pp. 1917-1923.

[33] M. Panfili, A. Pietrabissa, G. Oddi, V. Suraci, A lexicographic approach to constrained MDP admission control, International Journal of Control 89 (2016) 235-247.

[34] J. Perez, C. Germain-Renaud, B. Kégl, C. Loomis, Multi-objective reinforcement learning for responsive grids, Journal of Grid Computing 8 (2010) 473-492.

[35] N. Sprague, D. Ballard, Multiple-Goal Reinforcement Learning with Modular Sarsa(0), Technical Report 798, Computer Science Department, The University of Rochester, 2004.

[36] L. Yliniemi, K. Tumer, Multi-objective multiagent credit assignment through difference rewards in reinforcement learning, in: Simulated Evolution and Learning, Springer, 2014, pp. 407-418.

[37] K. Van Moffaert, A. Nowé, Multi-objective reinforcement learning using sets of pareto dominating policies, Journal of Machine Learning Research 15 (2014) 3483-3512.

[38] K. Van Moffaert, T. Brys, A. Nowé, Risk-sensitivity through multiobjective reinforcement learning, in: Evolutionary Computation (CEC), 2015 IEEE Congress on, IEEE, 2015, pp. 1746-1753.

[39] P. Vamplew, R. Dazeley, A. Berry, E. Dekker, R. Issabekov, Empirical evaluation methods for multiobjective reinforcement learning algorithms, Machine Learning 84 (2011) 51-80. 
[40] P. Vamplew, R. Issabekov, R. Dazeley, C. Foale, Reinforcement learning of pareto-optimal multiobjective policies using steering, in: AI 2015: Advances in Artificial Intelligence, Springer, 2015, pp. 596-608.

[41] P. Vamplew, R. Issabekov, R. Dazeley, C. Foale, A. Berry, T. Moore, D. Creighton, Steering approaches to pareto-optimal multiobjective reinforcement learning, Neurocomputing (this issue) (2016).

[42] W. Wang, M. Sebag, Multi-objective monte-carlo tree search, in: Asian Conference on Machine Learning, volume 25, 2012, pp. 507-522.

[43] W. Wang, M. Sebag, Hypervolume indicator and dominance reward based multi-objective monte-carlo tree search, Machine Learning 92 (2013) 403-429.

[44] M. Wiering, M. Withagen, M. M. Drugan, Model-based multi-objective reinforcement learning, in: Adaptive Dynamic Programming and Reinforcement Learning (ADPRL), 2014 IEEE Symposium on, IEEE, 2014, pp. 1-6.

[45] Y. Zhao, Q. Chen, W. Hu, Multi-objective reinforcement learning algorithm for MOSDMP in unknown environment, in: Proceedings of the 8th World Congress on Intelligent Control and Automation, 2010, pp. 3190-3194.

[46] E. Zitzler, J. Knowles, L. Thiele, Quality assessment of pareto set approximations, in: Multiobjective Optimization, Springer, 2008, pp. 373404.

[47] L. M. Zintgraf, T. V. Kanters, D. M. Roijers, F. A. Oliehoek, P. Beau, Quality assessment of MORL algorithms: A utility-based approach (2015).

[48] M. Gregor, J. Spalek, Curiosity-driven exploration in reinforcement learning, in: ELEKTRO, 2014, IEEE, 2014, pp. 435-440. 